University of Nebraska - Lincoln

DigitalCommons@University of Nebraska - Lincoln

Faculty Publications, Department of Physics and Astronomy

Research Papers in Physics and Astronomy

April 2002

\title{
Multielectron system in an ultrashort, intense laser field: A nonperturbative, time-dependent two-active-electron approach
}

\author{
G. Lagmago Kamta \\ University of Nebraska - Lincoln \\ Anthony F. Starace \\ University of Nebraska-Lincoln, astarace1@unl.edu
}

Follow this and additional works at: https://digitalcommons.unl.edu/physicsfacpub

Part of the Physics Commons

Kamta, G. Lagmago and Starace, Anthony F., "Multielectron system in an ultrashort, intense laser field: A nonperturbative, time-dependent two-active-electron approach" (2002). Faculty Publications, Department of Physics and Astronomy. 48.

https://digitalcommons.unl.edu/physicsfacpub/48

This Article is brought to you for free and open access by the Research Papers in Physics and Astronomy at DigitalCommons@University of Nebraska - Lincoln. It has been accepted for inclusion in Faculty Publications, Department of Physics and Astronomy by an authorized administrator of DigitalCommons@University of Nebraska Lincoln. 


\title{
Multielectron system in an ultrashort, intense laser field: A nonperturbative, time-dependent two-active-electron approach
}

\author{
G. Lagmago Kamta and Anthony F. Starace \\ Department of Physics and Astronomy, The University of Nebraska, 116 Brace Laboratory, Lincoln, Nebraska 68588-0111
}

(Received 12 December 2001; published 16 May 2002)

\begin{abstract}
We present a two-active-electron (TAE) approach for solving the time-dependent Schrödinger equation (TDSE) for the interaction of a multi-electron system with an ultrashort, intense, and linearly polarized laser pulse [Lagmago Kamta and Starace, Phys. Rev. Lett. 86, 5687 (2001)]. A technique for obtaining angular distributions for double ionization by such pulses is also described. The approach for solving the TDSE in the TAE approximation is full dimensional and accounts for correlations between the two electrons, as well as the polarization of the core. It is based on a configuration-interaction expansion of the time-dependent wave function in terms of one-electron atomic orbitals. Applying the method to the lithium negative ion $\left(\mathrm{Li}^{-}\right)$, we display the time-dependent dynamics of the photodetachment process. For low intensities, our results for the detachment yield follow expectations from lowest-order perturbation theory and agree satisfactorily with $\mathcal{R}$-matrix calculations. Our results for angular distributions indicate that following multiphoton double ionization by an intense laser field, electrons are predominantly ejected along the laser polarization axis; however, a significant number are ejected perpendicularly to this axis. An angular momentum-based analysis of these angular distributions indicates that, in the dipole approximation and for an initial ${ }^{1} S^{e}$ state interacting with a linearly polarized laser field, double ejection of both electrons along the direction perpendicular to the laser polarization axis can only occur following absorption of an even number of photons, whereas multiphoton absorption of an odd number of photons does not lead to double ejection at these angles.
\end{abstract}

DOI: 10.1103/PhysRevA.65.053418

PACS number(s): $32.80 . \mathrm{Rm}, 32.80 . \mathrm{Gc}, 32.80 . \mathrm{Wr}, 31.70 . \mathrm{Hq}$

\section{INTRODUCTION}

In studying theoretically the interaction of an atomic system with an ultrashort, intense laser pulse, the intense character of the field requires a nonperturbative approach, and the finite duration of the pulse calls for a direct numerical integration of the time-dependent Schrödinger equation (TDSE). Although a direct numerical integration of the TDSE is numerically intensive, for one-electron systems this is now a routine task on standard workstations and even PCs. However, for two-electron systems, which are the simplest multielectron systems, this remains a challenge, due to the high dimensionality of the problem (five dimensions for a linearly polarized laser field), which makes the numerical integration both highly time consuming and a drain on computer resources. This difficulty has led to the development of many approximations aiming at reducing the number of dimensions of the problem, either by treating model atoms in only one or two dimensions, or by treating only a single active electron (see, e.g., Refs. [1] and [2] for reviews). Only recently, owing to increases in computer speeds and memory capacities, have the $a b$ initio approaches to direct numerical solution of the TDSE for two-electron systems been developed [3-8]. However, all these approaches deal only with helium or $\mathrm{H}^{-}$.

For multielectron systems having more than two electrons, previous attempts to solve the TDSE for their interaction with an ultrashort, intense laser pulse have been essentially restricted to the single-active-electron (SAE) approximation. In the SAE approach [9], all electrons are assumed to be frozen and only one is allowed to interact with the laser field. The SAE has provided considerable insight into the single-electron response of multielectron systems to high-intensity laser excitation (see, e.g., Ref. [10] and references therein). However, many experiments [11-13] show evidence of nonsequential double ionization, thereby indicating the need for theory to go beyond the SAE approximation, i.e., to develop approaches that account for electron correlations. This quest for multielectron effects naturally suggests a two-active-electron (TAE) approximation as a first step, in which two electrons are allowed to respond to the laser excitation and to interact with each other. In this paper we describe such an approach, which is applicable to any multielectron system having two electrons outside one or more closed shells. The atomic system is treated approximately as a two-active electron system comprising the atomic core (which includes the nucleus and all inner-shell electrons) and the two outer electrons. Correlations between the two electrons are treated in our approach using the multipole expansion of the Coulomb repulsion between the two electrons, and interactions of the core with the two-active electrons are included via a pseudopotential, which accounts for the interactions of each electron with the core and for the effects of core polarization. Needless to say, our approach is applicable as long as the laser intensity it not high enough to influence the core electrons.

Angular distributions for double ionization of atomic systems by single photon impact have attracted much interest from both theorists and experimentalists (see, e.g., the reviews in Ref. [14]). In this case double ionization occurs only via correlation between the two electrons. Most ab initio theoretical approaches evaluate the triply differential cross section (TDCS) for single-photon double ionization using the transition matrix coupling the initial and final states [15]. 
Obtaining angular distributions for an ultrashort, intense laser pulse is challenging not only because the timedependent Schrödinger equation has to be solved in its full dimensionality, but also because the finiteness of the pulse introduces an uncertainty into the sharing of the excess energy. In fact, for multiphoton double ionization by intense fields, very little is known concerning the angular distributions of ejected electrons, despite the availability of COLTRIMS [13] and electron coincidence [16] measurements. On the theoretical side, although angle-averaged radial probability density plots (averaged over all angles except $\theta_{12}$ ) indicate that the two electrons may be ejected with a small relative angle $\theta_{12}$ by an ultrashort intense laser pulse [17], a technique for extracting detailed angular distributions is still lacking. In this paper, we propose such a technique, which allows one to obtain detailed angular distributions for double ionization following the solution of the TDSE. Brief reports on our theoretical approach and on our initial results for double ionization electron angular distributions have been presented elsewhere $[18,19]$.

As an application of our method described above, we consider the interaction of $\mathrm{Li}^{-}$with an ultrashort, intense laser field. Owing to the fact that $\mathrm{Li}^{-} 1 s^{2} 2 s^{2}\left({ }^{1} S\right)$ has an outer closed $s$ subshell, it may be regarded as the second simplest negative ion, differing from $\mathrm{H}^{-}$mainly by having a more extended core, consisting of a nucleus and two $1 s$ electrons. In this work, we are interested in the single and double photoionization of the $\mathrm{Li}^{-}$negative ion in its stable ground state, $1 s^{2} 2 s^{2}\left({ }^{1} S\right)$. Our choice of $\mathrm{Li}^{-}$is motivated by specific features related to our numerical approach, as well as by the availability of experimental and theoretical data for singlephoton detachment with which comparison is possible. Indeed, as discussed later, our calculations are done in a spherical radial box. In principle, the higher the laser intensity, the larger the box should be. Unfortunately, a larger box implies a longer computation time as well as larger computer memory requirements. Since the two outer electrons in $\mathrm{Li}^{-}$ have a low binding energy, the nonperturbative regime sets in at not too high laser intensities. Thus a significant double ionization probability can be obtained at fairly low intensities $\left(10^{11} \mathrm{~W} / \mathrm{cm}^{2}\right)$. This fact, in addition to the absence of a Rydberg spectrum in $\mathrm{Li}^{-}$, allows us to perform calculations with reasonable box sizes.

Photodetachment of $\mathrm{Li}^{-}$has attracted much interest, both theoretically and experimentally. Experiments have been performed [20-24] for the detachment from the ground state by absorption of one or more photons, either by a direct ejection or via a temporary negative ion resonance. On the theoretical side, since the early work of Moores and Norcross [25], accurate calculations of the single-photon detachment cross section of $\mathrm{Li}^{-}$for various energy domains have been performed using various approaches: the $\mathcal{K}$-matrix method [26], the $\mathcal{R}$-matrix method [27], the eigenchannel $\mathcal{R}$-matrix method [28,29], and a numerical basis set combined with the complex rotation method [30]. For the multiphoton case, calculations have been performed using the "state specific" [31] and the $\mathcal{R}$-matrix Floquet [32] approaches.

In this work, we perform a time-dependent study of both detachment and double ionization of $\mathrm{Li}^{-}$by an ultrashort, intense laser pulse. In the low-intensity regime, we show that our results agree with results of lowest-order-perturbation theory (LOPT), as well as with the $\mathcal{R}$-matrix Floquet calculations $[32,33]$. For a laser frequency just above the detachment threshold, we illustrate and discuss the channel closure that occurs in the detachment yield as the field peak intensity increases, due to ac Stark and ponderomotive shifts. We also obtain detailed angular distributions for single photon (low field intensity) and for multiphoton (nonperturbative field intensity) double ionization by ultrashort laser pulses. Our angular distribution results show new features (relative to those for a weak, monochromatic laser field) stemming from the intense character of the laser field, electron-electron correlations, as well as symmetry effects originating from Pauli exclusion principle and parity requirements.

This paper is organized as follows: In Sec. II, we discuss the basic assumptions of the TAE approximation and present the numerical approach used to solve the TDSE. The timedependent dynamics of the detachment process are illustrated in Sec. III. Results for the intensity dependence of the detachment yield are given in Sec. IV, where we also discuss channel closure effects. Angular distributions for twoelectron ionization of $\mathrm{Li}^{-}$are discussed in Sec. V. Section VI presents a summary of our results and our conclusions. Unless otherwise stated, atomic units (a.u.) are used throughout this work.

\section{SOLUTION OF THE TDSE FOR A TWO-ACTIVE ELECTRON SYSTEM}

We treat $\mathrm{Li}^{-}$as a two-active electron system, where each electron is assumed to move in the following pseudopotential describing the $\mathrm{Li}^{+}$core [34]:

$$
\begin{aligned}
V(r)= & -\frac{1}{r}\left[Z_{c}+\left(Z_{n}-Z_{c}\right) e^{-a_{1} r}+a_{2} r e^{-a_{3} r}\right] \\
& -\frac{\alpha_{c}}{2 r^{4}}\left(1-e^{-\left(r / r_{c}\right)^{3}}\right)^{2} .
\end{aligned}
$$

Here $Z_{c}$ and $Z_{n}$ denote the $\mathrm{Li}^{+}$core charge $\left(Z_{c}=1\right)$ and the nuclear charge $\left(Z_{n}=3\right)$, and $\alpha_{c}=0.1894$ is the polarizability of the $\mathrm{Li}^{+}$core [35]. The single-electron Hamiltonian for the interaction of each electron with the core is

$$
h(r)=-\frac{\boldsymbol{\nabla}_{r}^{2}}{2}+V(r) \text {. }
$$

It follows that the equation describing the radial motion of each electron, having angular momentum $\ell$, in the field of the core is given by

$$
\left(-\frac{1}{2} \frac{d^{2}}{d r^{2}}+\frac{\ell(\ell+1)}{2 r^{2}}+V(r)\right) R(r)=\mathcal{E} R(r) .
$$

The core potential parameters $a_{1}, a_{2}$, and $a_{3}$ in Eq. (1) are fitted such that solving Eq. (3) yields the experimentally measured energy levels of the Li atom [36]. Note that the resulting core potential has been used in time-independent 
eigenchannel $\mathcal{R}$-matrix calculations $[28,29]$ to predict photodetachment cross sections that agree with results of experimental measurements in the perturbative laser intensity regime.

The atomic Hamiltonian of $\mathrm{Li}^{-}$accounting for electron correlations is

$$
H=h\left(r_{1}\right)+h\left(r_{2}\right)+\frac{1}{r_{12}},
$$

where $r_{12}=\left|\mathbf{r}_{1}-\mathbf{r}_{2}\right|$ denotes the interelectron coordinate, while the labels 1 and 2 refer respectively to electrons 1 and 2. We employ the multipole expansion of the electrostatic interaction potential,

$$
\frac{1}{r_{12}}=\sum_{q=0}^{\infty} \frac{4 \pi}{2 q+1} \frac{r_{<}^{q}}{r_{>}^{q+1}} \sum_{p=-q}^{q} Y_{q, p}^{*}\left(\hat{r}_{<}\right) Y_{q, p}\left(\hat{r}_{>}\right),
$$

where $Y_{q, p}(\hat{r})$ denotes a spherical harmonic.

The TDSE equation describing the interaction of the atomic system with a linearly polarized laser field is

$$
i \frac{\partial}{\partial t} \Psi_{L, V}\left(\mathbf{r}_{1}, \mathbf{r}_{2}, t\right)=\left[H+D_{L, V}(t)\right] \Psi_{L, V}\left(\mathbf{r}_{1}, \mathbf{r}_{2}, t\right),
$$

where the operator $D_{L, V}(t)$ describes the interaction of the system with the laser field. In the dipole approximation, $D_{L, V}(t)$ is given by either $D_{L}(t)=\mathbf{E}(t) \cdot\left(\mathbf{r}_{1}+\mathbf{r}_{2}\right)$ or $D_{V}(t)$ $=\mathbf{A}(t) \cdot\left(\mathbf{p}_{1}+\mathbf{p}_{2}\right)$ in the length $(L)$ and velocity $(V)$ forms, respectively. For simplicity of notation, we will simply write $\Psi$ to denote the wave function in both gauges, and only specify the index ( $L$ or $V$ ) when it is relevant to do so. The electric field $\mathbf{E}(t)$ and the vector potential $\mathbf{A}(t)$ are given by

$$
\mathbf{E}(t)=-\frac{\partial}{\partial t} \mathbf{A}(t), \quad \mathbf{A}(t)=\hat{\mathbf{z}} A_{0} f(t) \sin \omega t,
$$

where $\omega$ is the laser frequency, $\hat{\mathbf{z}}$ is the unit vector along the linear polarization axis of the field, and $f(t)$ is the pulse envelope, which is assumed throughout this work to have the squared cosine form

$$
f(t)= \begin{cases}\cos ^{2}(t / \tau), & -\pi \tau / 2 \leqslant t \leqslant \pi \tau / 2 \\ 0, & \text { otherwise }\end{cases}
$$

We integrate the TDSE (6) in a box using the following CI expansion of the time-dependent wave function $\Psi(t)$,

$$
\begin{aligned}
\Psi\left(\mathbf{r}_{1}, \mathbf{r}_{2}, t\right)= & \sum_{L, M} \sum_{\ell_{1}, \ell_{2}} \sum_{n_{1}, n_{2}} \psi_{n_{1} n_{2}}^{\ell_{1} \ell_{2} L M}(t) \\
& \times \mathcal{A} \frac{R_{n_{1}, \ell_{1}}\left(r_{1}\right)}{r_{1}} \frac{R_{n_{2}, \ell_{2}}\left(r_{2}\right)}{r_{2}} \mathcal{Y}_{\ell_{1}, \ell_{2}}^{L, M}\left(\hat{r}_{1}, \hat{r}_{2}\right),
\end{aligned}
$$

where the set of constants $\psi_{n_{1} n_{2}}^{\ell_{1} \ell_{2} L M}$ are the expansion coefficients. $\mathcal{A}$ is the normalized antisymmetrization operator: $\mathcal{A}$ $=\left(1+\varepsilon P_{12}\right) / \sqrt{2}$, where $\varepsilon=+1$ (respectively -1 ) for singlet (respectively triplet) states. The operator $P_{12}$ simulta- neously exchanges the parameters $\left(n_{1}, \ell_{1}\right)$ and $\left(n_{2}, \ell_{2}\right)$ in order to account for the indistinguishability of the two electrons. Thus, $\mathcal{A}$ projects onto either singlet or triplet states, so as to assure the symmetry or the antisymmetry of the spatial wave function, in accordance with the Pauli exclusion principle. The bipolar spherical harmonics, which couple the individual angular momenta of the two electrons in the $L-S$ coupling scheme, are given by [37]

$$
\mathcal{Y}_{\ell_{1}, \ell_{2}}^{L, M}\left(\hat{r}_{1}, \hat{r}_{2}\right)=\sum_{m_{1} m_{2}} C_{\ell_{1} m_{1} \ell_{2} m_{2}}^{L M} Y_{\ell_{2}, m_{2}}\left(\hat{r}_{1}\right) Y_{\ell_{2}, m_{2}}\left(\hat{r}_{2}\right),
$$

where $C_{\ell_{1} m_{1} \ell_{2} m_{2}}^{L M}$ denotes a Clebsch-Gordan coefficient. The radial wave functions $R_{n, \ell}$ are obtained (for both $\mathcal{E} \leqslant 0$ and $\mathcal{E}>0$ ) by solving Eq. (3) in a radial box of size $r=r_{0}$, with boundary conditions $R_{n, \ell}\left(r_{0}\right)=0$, where $n$ labels the number of nodes of $R_{n, \ell}(r)$ within $r_{0}$. Note that both bound and continuum one-electron orbitals are included in the basis expansion, so that the resulting basis set is complete (except for truncation). In order to solve Eq. (3), it is transformed into a set of two coupled first-order differential equations, which are solved by combining a Runge-Kutta method [38] of order 4 with the Adams-Moulton predictor-corrector method [39]. The Runge-Kutta method is used for the solution at smaller radial distances, where the short-range character of $V(r)$ is prominent; at larger distances the predictor-corrector method is used. The resulting solutions $R_{n, \ell}(r)$ are stored at various consecutive radial points on the grid of radius $r_{0}$. It follows from Eq. (9) that the time-dependent wave function is subjected to boundary conditions at $r=r_{0}$ that are similar to that of $R_{n, \ell}(r)$, i.e., $\Psi\left(r_{1}, r_{2}=r_{0}, t\right)=\Psi\left(r_{1}=r_{0}, r_{2}, t\right)=0$. In order to minimize reflections of the probability flux that may occur at the boundaries of the box during time propagation, its size, the laser intensities, and the pulse durations are adjusted such that throughout the time propagation process, the wave function remains negligible at the edges of the radial box.

Considering (i) pure $L-S$ coupling for the two-active electrons, (ii) a linearly polarized laser field, and (iii) the fact that the ground state of $\mathrm{Li}^{-}$is ${ }^{1} S$, we can set $M=0$ in the expansion (9). In addition, due to limitations on data storage capacities, the expansion (9) has to be truncated by introducing cutoff values for $L, \ell_{1}, \ell_{2}, n_{1}$, and $n_{2}$. In practice, $L$ $=0,1,2, \ldots, L_{\max }$. For each selected $L$, a limited number (about 4 to 7 ) of partial waves [i.e., $\left(\ell_{1}, \ell_{2}\right)$ pairs] is included in the expansion. Finally, for each partial wave, $N_{1}$ radial functions are selected for electron 1 and $N_{2}$ for electron 2, i.e., $n_{1}=1,2, \ldots, N_{1}$ and $n_{2}=1,2, \ldots, N_{2}$. The results are considered as converged when they become stable with increasing $L_{\max }$, the number of partial waves, and $N_{1}$ and $N_{2}$. Note that the number of radial functions selected for electron 1 may be different from that for electron 2 [40]. As pointed out in Ref. [41], this asymmetry in $N_{1}$ and $N_{2}$ allows one to keep the basis size within reasonable limits. Indeed, in describing a process in which one electron is detached while the other is left in the ground state or a much lower energy excited level, $N_{1}$ and $N_{2}$ need not both be large. A large $N_{1}$ 


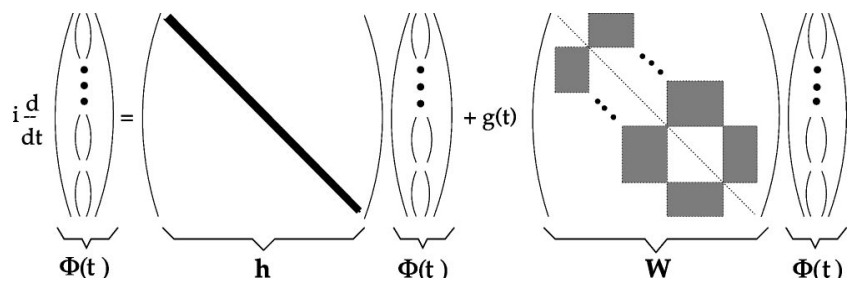

FIG. 1. Matrix structure of the TDSE (12) in the atomic basis. $\mathbf{h}$ is the diagonal matrix of atomic eigenvalues, and $\mathbf{W}$ is the dipole matrix in the atomic basis. $\mathbf{W}$ is sparse and has a band of offdiagonal blocks, in which each block corresponds to a pair $\left(L^{\prime}, L\right)$ of angular momenta satisfying $L^{\prime}-L= \pm 1$.

is necessary in the basis to describe the outgoing electron, while a smaller $N_{2}$ suffices to describe the inner electron.

The solution of the TDSE in the above basis is equivalent to solving the set of coupled first-order differential equations

$$
i \frac{\partial}{\partial t} \boldsymbol{\Psi}(t)=[\mathbf{H}+g(t) \mathbf{D}] \boldsymbol{\Psi}(t),
$$

where $\mathbf{H}$ and $\mathbf{D}$ are real matrices associated respectively with the atomic Hamiltonian and the dipole operator; $\mathbf{H}$ is symmetric, while $\mathbf{D}$ is symmetric in the length form and antisymmetric in the velocity form; $\boldsymbol{\Psi}(t)$ is a vector representing the wave function; and $g(t)$ is a scalar function that equals $E(t)$ for the length form of the dipole interaction, and $-i A(t)$ for the velocity form. $\mathbf{H}$ is sparse and has a block diagonal structure owing to the angular momentum selection rules $\Delta L$ $=L^{\prime}-L=0$, while $\mathbf{D}$ is also sparse but has a band structure in blocks owing to the electric dipole selection rules $\Delta L$ $=L^{\prime}-L= \pm 1$. Each bloc of $\mathbf{H}$ corresponds to a given $L$, whereas each bloc of $\mathbf{D}$ corresponds to a pair $\left(L^{\prime}, L\right)$. Equation (11) can be solved directly, using Runge-Kutta methods. However, we diagonalize $\mathbf{H}$ and project Eq. (11) onto the more convenient atomic eigenstate representation or atomic basis $[4,42]$, where it becomes

$$
i \frac{\partial}{\partial t} \boldsymbol{\Phi}(t)=[\mathbf{h}+g(t) \mathbf{W}] \boldsymbol{\Phi}(t),
$$

where $\boldsymbol{\Phi}(t)=\mathbf{P}^{t} \boldsymbol{\Psi}(t), \mathbf{P}$ is the orthogonal matrix of eigenvectors of $\mathbf{H}$, and $\mathbf{P}^{t}$ is its transpose (i.e., $\mathbf{P}^{t} \mathbf{P}=\mathbf{1}$, where $\mathbf{1}$ is the unit matrix). The matrix structure of Eq. (12) is shown in Fig. 1. Here $\mathbf{h}=\mathbf{P}^{t} \mathbf{H P}$ represents the diagonal matrix of eigenvalues of $\mathbf{H}$, and $\mathbf{W}=\mathbf{P}^{t} \mathbf{D P}$ the dipole matrix elements coupling various two-electron eigenstates. $\mathbf{W}$ has the same band structure as $\mathbf{D}$. The resulting wave function $\boldsymbol{\Phi}(t)$ in the atomic basis represents a linear superposition of two-electron eigenstates resulting from the diagonalization, i.e.,

$$
\boldsymbol{\Phi}(t)=\sum_{n, L} C_{n, L} \boldsymbol{\Phi}_{n, L}
$$

where $\boldsymbol{\Phi}_{n, L}$ is a two-electron eigenstate of energy $E_{n}$ and $C_{n, L}$ is its probability amplitude. Note that $\boldsymbol{\Psi}(t)$ can be easily deduced from $\boldsymbol{\Phi}(t)$ by the matrix vector product $\boldsymbol{\Psi}(t)$ $=\mathbf{P \Phi}(t)$. The last transformation allows one to move back and forth between the eigenstate and the coordinate represen-

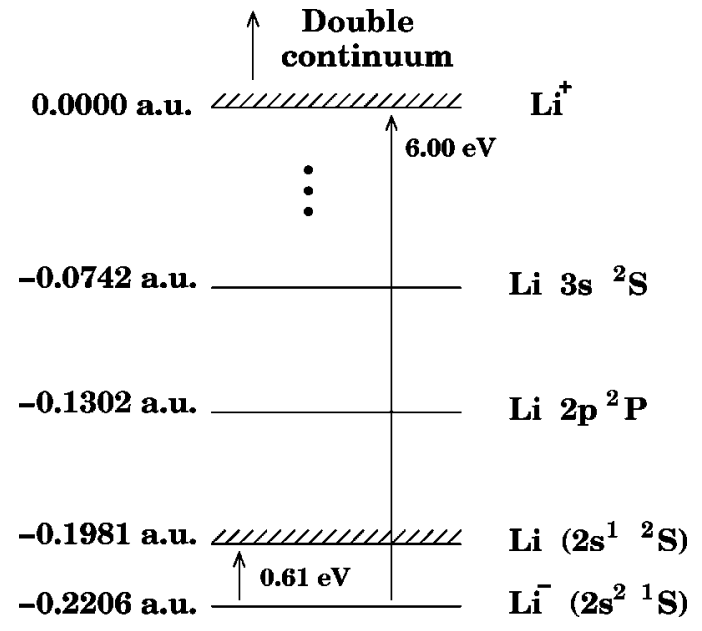

FIG. 2. Energy level diagram showing the ground state of $\mathrm{Li}^{-}$, the ground state and first two excited states of $\mathrm{Li}$, and the ground state of $\mathrm{Li}^{+}$(which is taken as the zero of the energy scale). Energies corresponding to the levels are given in the left column in atomic units (a.u.). About $0.61 \mathrm{eV}$ are necessary to eject one electron from $\mathrm{Li}^{-}$, and about $6.00 \mathrm{eV}$ are necessary to eject two electrons.

tations. We solve the TDSE (12) by using an embedded Runge-Kutta method [43] of order 5. An important parameter to control in the atomic basis is the density of two-electron atomic states [7]. Two parameters allow us to control this density here: the box size and the number of one-electron radial functions included in the expansion.

The results presented in this work are obtained with $L_{\max }=8$ and a spherical box of radius $r_{0}=250$ a.u. The number of configurations for each total angular momentum $L$ varies between 2700 and 3600 , leading to a system of at least 20000 ordinary differential equations to be solved. A typical time propagation runs for about $24 \mathrm{~h}$ (or longer with increasing laser intensity and pulse duration) on a $660 \mathrm{MHz}$ DEC workstation with $1 \mathrm{~Gb}$ of random access memory. We have varied the size of the box from 180 a.u to 300 a.u. as well as the number of angular momenta to check the stability of our results. The binding energy obtained for $\mathrm{Li}^{-}$is $E_{g}$ $=-0.02251$ a.u. $(0.6125 \mathrm{eV})$, which is in good agreement with the measured value, -0.02269 a.u. $(0.6174 \mathrm{eV})$ [44]. A diagram showing some energy levels of $\mathrm{Li}^{-}$obtained from our calculations is given in Fig. 2. Throughout this work, the initial state for the time-propagation is the ground state of the system.

\section{PHOTODETACHMENT DYNAMICS OF $\mathrm{Li}^{-}$}

The population in any eigenstate $\boldsymbol{\Psi}_{\alpha}$ of $\mathbf{H}$ can be monitored during the interaction of the system with the laser field by evaluating the projection $P_{\alpha}(t)=\left|\left\langle\boldsymbol{\Psi}_{\alpha} \mid \boldsymbol{\Psi}_{L}(t)\right\rangle\right|^{2}$, where $\boldsymbol{\Psi}_{L}(t)$ is the solution of the TDSE at time $t$ in the length gauge. In fact, in the length gauge, the projection of the time-dependent wave function onto a field-free state can be interpreted as a probability amplitude [45], whereas in the velocity gauge, one should alternatively use $P_{\alpha}(t)$ $=\left|\left\langle\boldsymbol{\Psi}_{\alpha} \mid \exp \left[-i \mathbf{A}(t) \cdot\left(\mathbf{r}_{1}+\mathbf{r}_{2}\right)\right] \boldsymbol{\Psi}_{V}(t)\right\rangle\right|^{2}$, which is numeri- 


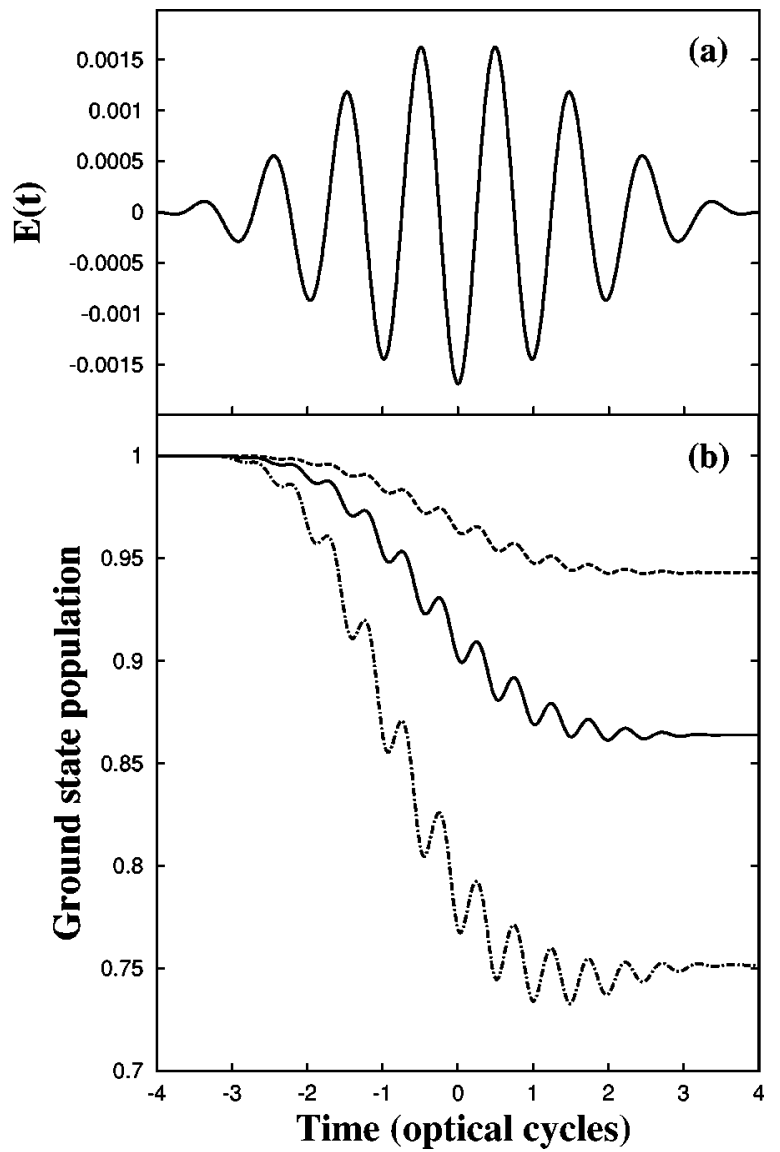

FIG. 3. Time evolution of the ground-state population of $\mathrm{Li}^{-}$in the presence of an ultrashort laser pulse of frequency $\omega$ $=0.024$ a.u. $(1898.5 \mathrm{~nm})$ containing four cycles at FWHM. The time on the horizontal axis in given in units of the laser period, $2 \pi / \omega$. (a) Time dependence of the laser electric field $E(t)$ for a peak intensity $I=1 \times 10^{11} \mathrm{~W} / \mathrm{cm}^{2}$. (b) Time dependence of the ground-state population for laser peak intensities $I=1$ $\times 10^{10} \mathrm{~W} / \mathrm{cm}^{2}$ (upper plot), $I=5 \times 10^{10} \mathrm{~W} / \mathrm{cm}^{2}$ (middle plot), $I$ $=1 \times 10^{11} \mathrm{~W} / \mathrm{cm}^{2}$ (lower plot).

cally cumbersome to evaluate in our case. The time evolution of $P_{\alpha}(t)$ for the ground state provides a qualitative insight into the detachment dynamics. Figure 3(b) displays the time evolution of the ground-state population of $\mathrm{Li}^{-}$for various peak intensities $\left(10^{10}, 5 \times 10^{10}\right.$, and $\left.10^{11} \mathrm{~W} / \mathrm{cm}^{2}\right)$ for a laser pulse of frequency $\omega=0.024$ a.u. $(1898.5 \mathrm{~nm})$, containing 4 cycles within the full width at half maximum (FWHM), which corresponds to a total of 8 laser cycles in the pulse. The electric field corresponding to this laser pulse is shown in the top panel [Fig. 3(a)] to guide the eye. It appears that the ground-state population oscillates with the laser field and is significantly depleted as the laser peak intensity increases. During each half cycle when the laser field magnitude reaches a maximum, a burst of population leaves the ground state. Note that for a peak intensity of only 5 $\times 10^{10} \mathrm{~W} / \mathrm{cm}^{2}$, almost $15 \%$ of the ground-state population is depleted, indicating that even at such a fairly low intensity the behavior of $\mathrm{Li}^{-}$is already nonperturbative. Indeed, owing to the fact that $\mathrm{Li}^{-}$is a loosely bound system, nonperturbative behavior sets in at relatively low laser intensities.

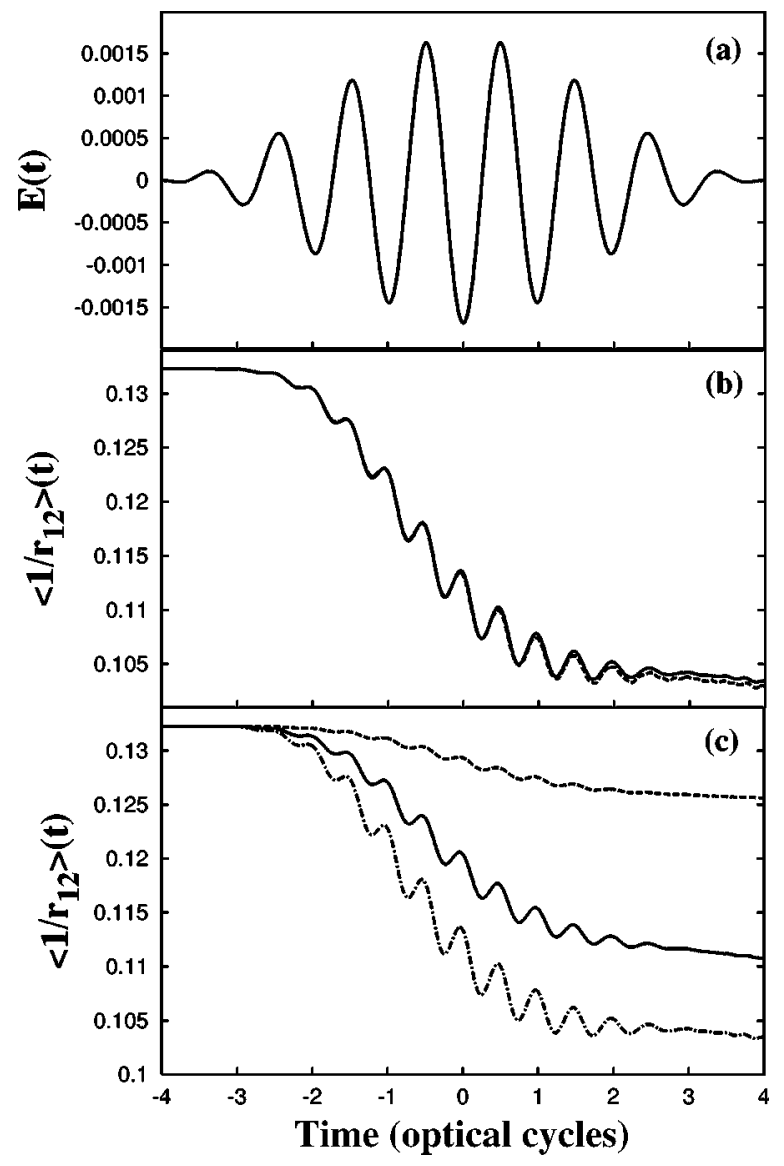

FIG. 4. Time evolution of the Coulomb repulsion between the two electrons in $\mathrm{Li}^{-}$interacting with an ultrashort laser pulse of frequency $\omega=0.024$ a.u. $(1898.5 \mathrm{~nm})$ containing four cycles at FWHM. The time on the horizontal axis is given in units of the laser period, $2 \pi / \omega$. (a) Time dependence of the laser electric field $E(t)$ for a peak intensity $I=1 \times 10^{11} \mathrm{~W} / \mathrm{cm}^{2}$. (b) Time dependence of the averaged Coulomb repulsion between the two electrons for a field intensity $I=10^{11} \mathrm{~W} / \mathrm{cm}^{2}$ in both the velocity gauge (solid line) and the length gauge (dashed line). (c) Comparison of the time dependence of the averaged Coulomb repulsion between the two electrons for three peak field intensities: $I=10^{10} \mathrm{~W} / \mathrm{cm}^{2}$ (upper plot), $I=5 \times 10^{10} \mathrm{~W} / \mathrm{cm}^{2}$ (middle plot), $I=1 \times 10^{11} \mathrm{~W} / \mathrm{cm}^{2}$ (lower plot).

In contrast to $P_{\alpha}(t)$, the expression

$$
\left\langle 1 / r_{12}\right\rangle(t)=\left\langle\Psi\left(\mathbf{r}_{1}, \mathbf{r}_{2}, t\right)\left|\frac{1}{r_{12}}\right| \Psi\left(\mathbf{r}_{1}, \mathbf{r}_{2}, t\right)\right\rangle
$$

which represents the mean value of the Coulomb repulsion at time $t$, is gauge independent and provides insight into the relative averaged dynamics of the two-active electrons in the presence of the laser field. For the laser peak intensity of

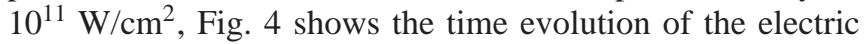
field [Fig. 4(a)], together with that of the Coulomb repulsion $\left\langle 1 / r_{12}\right\rangle(t)$ between the two electrons [Figs. 4(b) and 4(c)]. Results for $\left\langle 1 / r_{12}\right\rangle(t)$ obtained with the length and the velocity forms of the dipole operator agree well throughout the time of interaction with the laser field [see Fig. 4(b)]. This is 

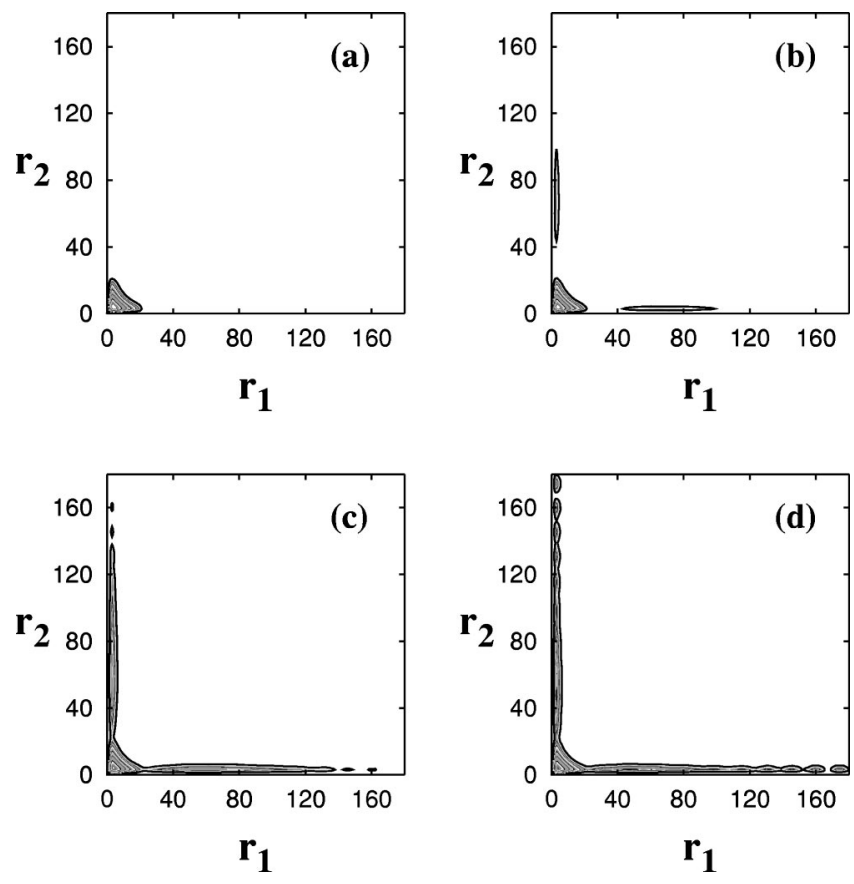

FIG. 5. Angle-integrated radial probability distributions: (a) for the ground state (before the laser pulse is switched on); (b), (c), and (d) for the wave function at the end of laser excitation for peak intensities $10^{10} \mathrm{~W} / \mathrm{cm}^{2}$ (a), $5 \times 10^{10} \mathrm{~W} / \mathrm{cm}^{2}$ (b) and $10^{11} \mathrm{~W} / \mathrm{cm}^{2}$ (c), respectively. The laser frequency and FWHM are the same as in Fig. 4.

a significant confirmation of the accuracy of our results. Figure 4 indicates that the Coulomb repulsion between the two electrons oscillates with the laser field and decreases every half cycle. This decrease is due primarily to the photodetachment of one electron. Indeed, as one electron is photodetached, with the other remaining bound, a probability flux associated with the detached electron is driven to larger distances, leading to a larger averaged interelectronic distance, and the Coulomb repulsion between the two electrons consequently decreases. As the laser field oscillates, the Coulomb repulsion experiences a local minimum and maximum during each half cycle. In fact, each half cycle, the electric field accelerates the outer electron to a larger distance up to a maximum, which corresponds to a local (in time) minimum in $\left\langle 1 / r_{12}\right\rangle(t)$; then the electric field changes sign and accelerates the outer electron towards the inner electron [leading to a local maximum in $\left.\left\langle 1 / r_{12}\right\rangle(t)\right]$. The overall decrease in the averaged Coulomb repulsion is also an indication that single-electron ejection dominates over double ejection, as indicated also by other evidence (see below). The averaged Coulomb repulsion between the two electrons is plotted in Fig. 4(c) for various laser peak intensities; the laser frequency and FWHM remaining the same. It is clear that the Coulomb repulsion between the two electrons decreases as the laser peak intensity increases.

Figure 5 displays the angle-integrated radial probability distributions for both the initial wave function (i.e., the ground state) [Fig. 5(a)] and for the wave function at the end of laser excitation for laser peak intensities of $10^{10} \mathrm{~W} / \mathrm{cm}^{2}$ [Fig. 5(b)], $5 \times 10^{10} \mathrm{~W} / \mathrm{cm}^{2}$ [Fig. 5(c)], and $10^{11} \mathrm{~W} / \mathrm{cm}^{2}$ [Fig. $5(\mathrm{~d})]$. Note the symmetry of the plots with respect to the exchange of the radial coordinates $r_{1}$ and $r_{2}$, due to the indistinguishability of the two electrons. Note also in Fig. 5(a) the large radial extent of the $\mathrm{Li}^{-}$ground state (up to distances of about 20 a.u. from the nucleus). A comparison of the figures indicates that after the laser excitation, a larger probability distribution is found along the $r_{1}$ and $r_{2}$ axes as the laser intensity increases. That the range of the outer electron probability distribution increases with the peak intensity is in accordance with the decrease in the Coulomb repulsion mentioned above. The fact that the distribution is essentially located along the axes indicates the dominance of the single ionization process.

\section{THE DETACHMENT YIELD OF $\mathrm{Li}^{-}$}

The detachment yield of $\mathrm{Li}^{-}$can be defined as

$$
D_{y}=1-\left|\left\langle\Psi_{0}\left(\mathbf{r}_{1}, \mathbf{r}_{2}\right) \mid \Psi\left(\mathbf{r}_{1}, \mathbf{r}_{2}, T\right)\right\rangle\right|^{2},
$$

where $\Psi_{0}\left(\mathbf{r}_{1}, \mathbf{r}_{2}\right)$ and $\Psi\left(\mathbf{r}_{1}, \mathbf{r}_{2}, T\right)$ respectively denote the initial ground-state wave function and the solution of the TDSE at time $T$ (marking the end of laser excitation). Table I presents the detachment yield for various peak intensities and frequencies for a laser field having 14 cycles FWHM, which corresponds to a total of about 28 laser cycles in the pulse. As in Refs. $[4,7]$ for $\mathrm{H}^{-}$, the $\mathcal{R}$-matrix Floquet (RMF) detachment yield is obtained by integrating the corresponding intensity-dependent rates [32,33] with the pulse shape used in our calculations. The agreement between our results and the RMF yield is better for low intensities, and disagrees increasingly with increasing intensity. Nevertheless, considering the presence of a pulse in our calculation, the timeindependent nature of RMF calculations, together with the

TABLE I. Detachment yield for various laser peak intensities $(I)$ and frequencies $(\omega)$, for a pulse duration of 14 optical cycles at FWHM in each case. The $\mathcal{R}$-matrix Floquet (RMF) yield is obtained by integrating the intensity-dependent RMF rate over the pulse envelope.

\begin{tabular}{cccccc}
\hline \hline $\mathrm{I}\left(\mathrm{W} / \mathrm{cm}^{2}\right)$ & $\omega($ a.u. $)$ & $\lambda(\mathrm{nm})$ & RMF rate [32] & RMF yield & Present \\
\hline $10^{9}$ & 0.0130 & 3504.9 & $8.66(-8)$ & 0.00038 & 0.00039 \\
$10^{9}$ & 0.0180 & 2531.3 & $5.67(-8)$ & 0.00027 & 0.00020 \\
$10^{10}$ & 0.0132 & 3451.8 & $8.19(-6)$ & 0.053 & 0.037 \\
$10^{10}$ & 0.0180 & 2531.3 & $5.58(-6)$ & 0.027 & 0.015 \\
\hline \hline
\end{tabular}




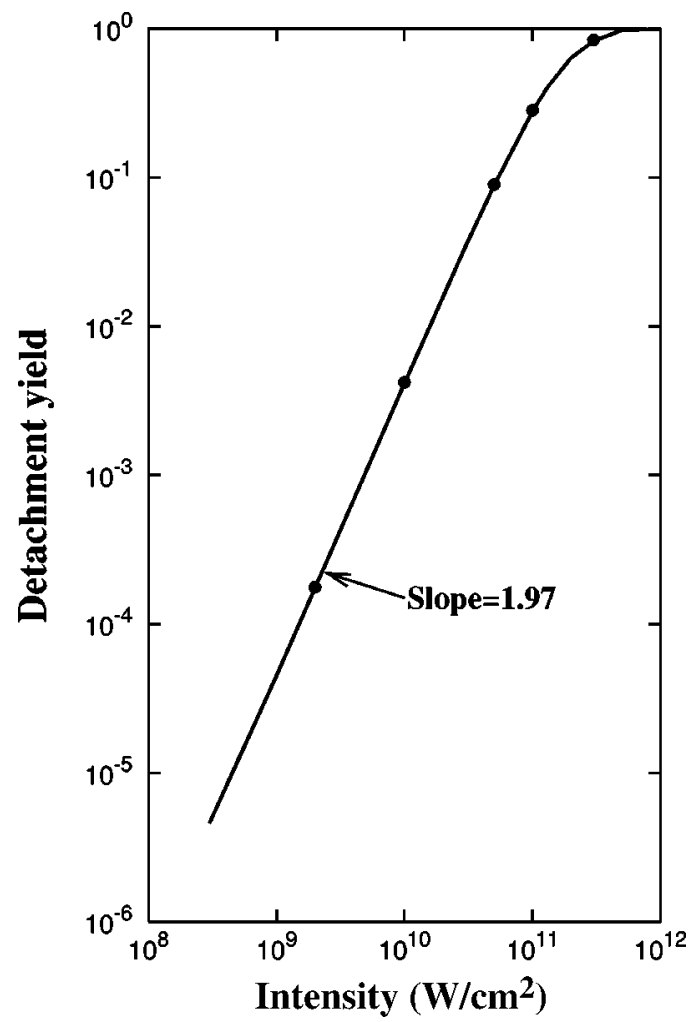

FIG. 6. Detachment yield for $\mathrm{Li}^{-}$as a function of the peak intensity of a laser pulse of frequency $\omega=0.018$ a.u. $(2531.3 \mathrm{~nm})$ having four cycles at FWHM: the solid line corresponds to the velocity form and the filled circles to the length form results.

fact that the above integration over the pulse shape is just an approximation, the agreement between the two approaches is rather satisfactory.

The photodetachment yield with respect to the laser peak intensity is plotted on a log-log scale in Figs. 6 and 7 for the frequencies $\omega=0.018$ a.u. (i.e., $2531.3 \mathrm{~nm}$ ) and $\omega$ $=0.024$ a.u. (i.e., $1898.5 \mathrm{~nm}$ ), respectively (see energy level diagram in Fig. 2). The total duration of the pulse considered for both cases is 8 cycles ( 4 cycles within FWHM), which leads to a FWHM pulse duration of about 34 fsec for $\omega$ $=0.018$ a.u., and about 25 fsec for $\omega=0.024$ a.u. For both frequencies, the photodetachment yield obtained using the length form (circles) and the velocity form (lines) of the dipole operator are in very close agreement. At low intensities, results in Figs. 6 and 7 indicate a linear dependence of the detachment yield with respect to the peak intensity of the laser pulse. The slope of this linear trend is about 1.99 for $\omega=0.018$ a.u., and 0.99 for $\omega=0.024$ a.u., in agreement with the LOPT. Indeed, according to LOPT, the $N$-photon absorption rate is given by $R_{N}=\sigma_{N} I^{N}$, where $I$ denotes the field intensity and $\sigma_{N}$ the generalized cross section. Therefore, in a log-log plot, the rate depends linearly with intensity with a slope equal to $N$. The frequency $\omega=0.024$ a.u. (0.653 $\mathrm{eV}$ ) is slightly above the detachment threshold, so that one photon is sufficient to photodetach at low intensities, leading to the slope $N \approx 1$ found in our results. On the other hand, the frequency $\omega=0.018$ a.u. $(0.490 \mathrm{eV})$ is below the detachment threshold so that at least two photons are necessary to

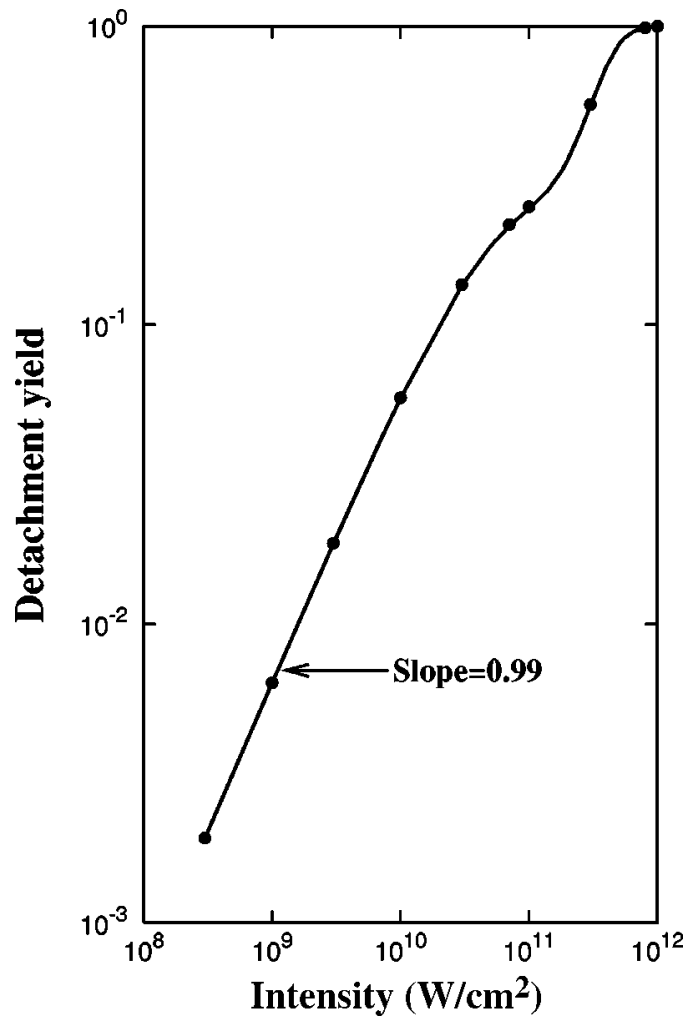

FIG. 7. Detachment yield for $\mathrm{Li}^{-}$as a function of the peak intensity of a laser pulse of frequency $\omega=0.024$ a.u. $(1898.5 \mathrm{~nm})$ having four cycles at FWHM: the solid line corresponds to the velocity form and the filled circles to the length form results.

photodetach, leading to the slope $N \approx 2$, which is also found in our results. With increasing intensity, the deviation of the yield from a linear dependence illustrates the breakdown of LOPT due to ac Stark and ponderomotive shifts, as well as higher-order effects. For both frequencies, saturation occurs rapidly for intensities of about $10^{12} \mathrm{~W} / \mathrm{cm}^{2}$, as the detachment yield becomes very close to 1 . However, for the frequency $\omega=0.024$ a.u., which lies just above the detachment threshold, an appreciable inflection appears in the yield before saturation occurs. This inflection is due to a combination of two intensity-related effects: The gradual closure of the onephoton detachment channel and the increase in the rate for the two-photon channel. Indeed, with increasing intensity, the dynamic Stark shift and the ponderomotive shift increase the energy gap between the ground state and the detachment threshold, leading to a closure with increasing laser intensity of the one-photon detachment channel, while increasing the rate for two-photon detachment.

Note that under similar conditions, the occurrence of a similar inflection in the detachment yield of $\mathrm{H}^{-}$has attracted interest recently. The authors of Ref. [6], in contrast with those of Ref. [4], did not find any inflection in their calculations for $\mathrm{H}^{-}$. Using two different approaches, subsequent calculations [7] confirmed this inflection. Its absence in the results of Ref. [6] may be due to their inaccurate value for the ground-state energy of $\mathrm{H}^{-}$, which leads to an incorrect position for the threshold [46,7].

To study the inflection in Fig. 7 in more detail, we have 

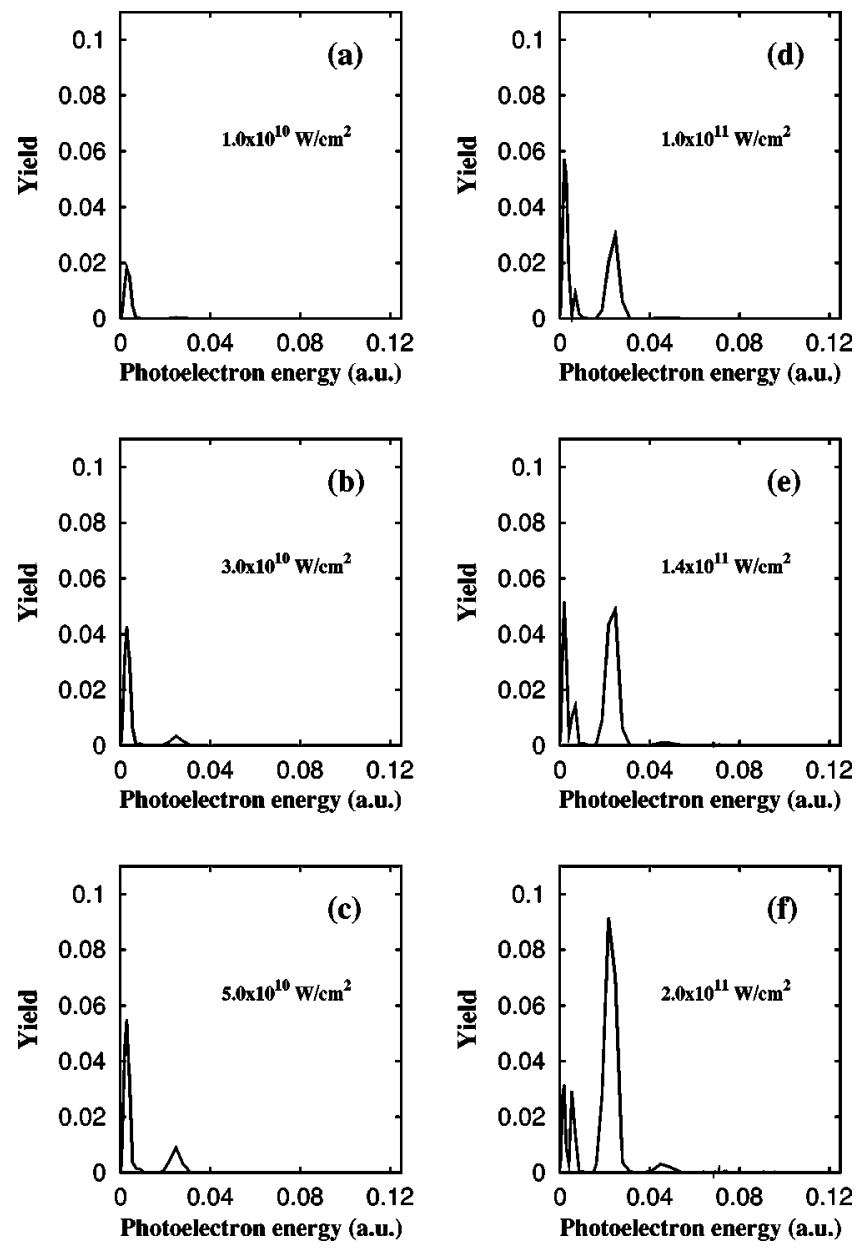

FIG. 8. Above-threshold detachment (ATD) spectra of $\mathrm{Li}^{-}$for various peak intensities corresponding to the inflection in the detachment rate shown in Fig. 7: (a) $I=10^{10} \mathrm{~W} / \mathrm{cm}^{2}$; (b) $I=3$ $\times 10^{10} \mathrm{~W} / \mathrm{cm}^{2}$; (c) $I=5 \times 10^{10} \mathrm{~W} / \mathrm{cm}^{2}$; (d) $I=1.0 \times 10^{11} \mathrm{~W} / \mathrm{cm}^{2}$; (e) $I=1.4 \times 10^{11} \mathrm{~W} / \mathrm{cm}^{2}$; (f) $I=2.0 \times 10^{11} \mathrm{~W} / \mathrm{cm}^{2}$. The laser frequency and FWHM are the same as in Fig. 7.

performed an energy analysis of the photodetached electron for intensities corresponding to the region where the bend occurs. Figure 8 gives the ATD (above-threshold detachment) spectra, i.e., the yield of electrons with respect to their energy for various peak intensities, and for a FWHM and frequency that are the same as those used to obtain the results in Fig. 7. As expected, with increasing laser peak intensity, more peaks, separated by the laser frequency, appear in the ATD spectrum. For convenience in discussing these results below, we present these plots on a linear scale rather than on the usual logarithmic scale. Consequently, we can focus only on the first few ATD peaks. Note that for each peak of the ATD spectrum, the area between the curve and the horizontal axis gives approximately the yield of electrons ejected following absorption of the corresponding number of photons. The spectrum in Fig. 8 (a) for $I=10^{10} \mathrm{~W} / \mathrm{cm}^{2}$ displays a single prominent peak corresponding to the absorption of a single photon above the detachment threshold. When the intensity is increased to $I=3 \times 10^{10} \mathrm{~W} / \mathrm{cm}^{2}[\mathrm{Fig}$. $8(\mathrm{~b})]$ and further to $I=5 \times 10^{10} \mathrm{~W} / \mathrm{cm}^{2}$ [Fig. 8(c)], a second peak corresponding to two-photon absorption appears in the
ATD spectrum. Note the steady increase in the height of the first ATD peak as the peak intensity is increased [cf. Figs. $8(\mathrm{a}), 8(\mathrm{~b})$, and $8(\mathrm{c})$ ], illustrating the increasing yield of electrons ejected following single-photon absorption. However, as the intensity is increased further, i.e., from 5 $\times 10^{10} \mathrm{~W} / \mathrm{cm}^{2}$ [Fig. 8(c)] to $10^{11} \mathrm{~W} / \mathrm{cm}^{2}$ [Fig. 8(d)] and then to $1.4 \times 10^{11} \mathrm{~W} / \mathrm{cm}^{2}$ [Fig. $\left.8(\mathrm{e})\right]$, which correspond to intensities in the region where the inflection in the yield is found in Fig. 7, one notes that the height of the first ATD peak remains at first unchanged and then begins to decrease. This indicates a decrease of the single-photon contribution to the detachment rate with increasing laser intensity, and coincides with a change in the slope of the intensity dependence of the detachment yield, appearing as the inflection in Fig. 7. Meanwhile, the contribution from the two-photon process increases substantially and is almost as important as that of the single-photon process. The situation is completely reversed in Fig. 8(f), which corresponds to the intensity 2 $\times 10^{11} \mathrm{~W} / \mathrm{cm}^{2}$ (which is located to the right of the inflection in Fig 7). At this intensity, the contribution from the twophoton process dominates over that of the single-photon process. The contribution from the single-photon detachment process drops substantially due to channel closure, which, because of the shortness of the pulse, is gradual rather than abrupt. After the inflection in Fig. 7, the intensity dependence of the yield experiences a sharp increase (before saturation), stemming from the contribution of two-photon absorption to the yield, whose intensity dependence is proportional to $I^{2}$ (in the LOPT picture).

Another feature seen in Fig. 8 is the splitting of the lowest ATD peak for intensities above $10^{11} \mathrm{~W} / \mathrm{cm}^{2}$ [cf. Fig. 8(d)8(f)]. Structures in ATI peaks have been observed experimentally in $\mathrm{Xe}[47,48]$ as well as in a number of timedependent theoretical calculations for both single-electron, one-dimensional model potentials [49-51] and for a fully correlated, two-electron model treated in three dimensions [6]. However, none of the structures found in the previous studies corresponds in detail to what our results show in Figs. 8(d)-8(f). Specifically, the experimental results observed structures that are attributed to multiphoton resonance transitions $[47,48]$ as well as to the ionic core fine-structure splitting [48]. Moreover, these structures are observed in all ATI peaks, although they are most prominent in the lowest energy ones. The one-dimensional theoretical calculations attribute the structures observed in all peaks to either resonance enhancement effects $[49,50]$ or to resonances between Floquet states [51]. The results of the realistic timedependent calculations for the $\mathrm{H}^{-}$ion find, in contrast to previous works, that a splitting occurs only in the highest energy ATI peak for a given laser intensity, and is attributed to a multiphoton threshold effect [6].

In contrast to these prior works, the splitting we observe occurs only in the lowest-energy ATD peak and the relative magnitude of the two subpeaks is intensity dependent. Relevant parameters of our calculation are as follows: the photon energy is 0.0240 a.u. $(0.653 \mathrm{eV}$ or $1898.5 \mathrm{~nm})$; the FWHM of our laser pulse is 1047 a.u., which corresponds to an energy spread (using the uncertainty relation) of about 0.001 a.u. $(0.027 \mathrm{eV})$; the ponderomotive potential $U_{p}$ 
equals 0.0012 a.u. $(0.033 \mathrm{eV})$ at peak intensity $10^{11} \mathrm{~W} / \mathrm{cm}^{2}$ and equals 0.0025a.u. (0.068 eV) at peak intensity 2 $\times 10^{11} \mathrm{~W} / \mathrm{cm}^{2}$; finally, the kinetic energy of the detached electron in the weak-field case is 0.0016 a.u. $(0.044 \mathrm{eV})$. Clearly, then, both the energy spread of the laser pulse and the magnitude of the ponderomotive potential at the peak intensity of the pulse are comparable in magnitude to the kinetic energy of the ionized (or, attempting to ionize) electron. The time-dependent ponderomotive potential associated with the ultrashort laser pulse permits single-photon detachment during the turning on and turning off of the pulse, but prevents single-photon detachment as the laser pulse approaches its maximum amplitude. We have carried out the following numerical experiment (not shown): we have investigated the ATD spectrum as a function of time during the course of the laser pulse. We find that as the pulse begins to increase from zero amplitude there is only a single ATD peak corresponding to absorption of a single photon. This single peak increases in magnitude and becomes narrower in energy as the laser pulse approaches its peak amplitude (the second ATD peak corresponding to the two-photon detachment also appears). Just past the peak amplitude, the first ATD peak divides into a strong narrow peak just above threshold and a higher-energy, weak, second peak; both peaks occupy the energy region of the initial single peak that we found to be produced at the beginning of the laser pulse. The weak, second peak increases in magnitude, while the strong, first peak decreases in magnitude, both to their final value at the end of the laser pulse (shown in Fig. 8). Thus, this numerical experiment indicates that the splitting occurs when the laser pulse reaches its peak amplitude, which gives an instantaneous ponderomotive potential which is comparable in magnitude to the kinetic energy of the electron above the singlephoton ionization threshold. The reasons why the initial ATD peak narrows and shifts to lower energies during the rise of the pulse and why the second peak (at higher energy) only appears during the second half of the laser pulse require further investigation.

\section{ANGULAR DISTRIBUTIONS FOR DOUBLE IONIZATION}

\section{A. Doubly differential double ionization probability}

Explicitly or implicitly, the time propagation of the TDSE for a finite pulse duration is always performed over finite distances. In this case, there is no exact definition for the double-ionization probability (DIP) owing to the difficulty of disentangling single- and double-ionization contributions from the time-propagated wave function. However, to obtain the double-ionization probability $P$ in intense pulsed-field calculations, one usually integrates the total probability found in the region $S_{3}$ in Fig. 9, which means evaluating the integral [52]

$$
P=\int d \Omega_{1} \int d \Omega_{2} \int_{r_{1}>r_{c}} d r_{1} \int_{r_{2}>r_{c}} d r_{2}\left|\Psi\left(\mathbf{r}_{1}, \mathbf{r}_{2}, T\right)\right|^{2},
$$

where $\mathbf{r}_{j} \equiv\left(r_{j}, \theta_{j}, \phi_{j}\right)$ and $d \Omega_{j} \equiv \sin \theta_{j} d \theta_{j} d \phi_{j}(j=1,2)$ denote

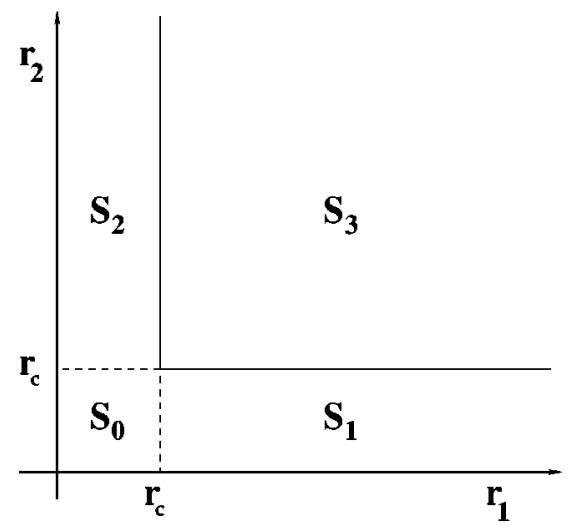

FIG. 9. Spatial grid in the radial coordinates $r_{1}$ and $r_{2}$ of the two electrons.

respectively the coordinates and the differential solid angles for the two electrons. $\Psi\left(\mathbf{r}_{1}, \mathbf{r}_{2}, T\right)$ represents the antisymmetrized wave function at time $T$, the end of the laser excitation. Angular integrations in Eq. (16) are performed over all angles (i.e., $0 \leqslant \theta_{j} \leqslant \pi, 0 \leqslant \phi_{j} \leqslant 2 \pi$ ), while radial integrations involve only configurations where the two electron radial coordinates are both larger than a cutoff radius $r_{c}$. The cutoff radius $r_{c}$ depends on the system under consideration, and should be chosen such that the region $S_{0}$ contains most of the probability distribution of the initial ground state. In our calculations, we use $r_{c}=20$ a.u., which is in accordance with the radial probability distribution for the ground state of $\mathrm{Li}^{-}$, as shown in Fig. 5(a). Note also that the populations in the regions $S_{1}$ and $S_{2}$ are attributed to single ionization. Of course, the definition (16) of the DIP is not exact because, for example, residual bound states and doubly excited states may well extend into the region $S_{3}$. These spurious contributions may be small, but they are nevertheless not negligible compared to the double-ionization probability, which is usually small as well. We also consider Eq. (16) as our definition of DIP, but with the difference that in our wave function $\Psi\left(\mathbf{r}_{1}, \mathbf{r}_{2}, T\right)$ [which is used for obtaining the DIP using Eq. (16)] we exclude any spurious contributions arising from populations left, at the end of time propagation, in atomic states below the double-ionization threshold (DIT). We thereby exclude any contributions from bound and doubly excited states, as well as singly excited states below the DIT. To exclude these spurious contributions, we proceed as follows: Let $\boldsymbol{\Phi}(T)$ be the solution of the TDSE (12) at time $T$. When deriving $\boldsymbol{\Psi}(T) \equiv \Psi\left(\mathbf{r}_{1}, \mathbf{r}_{2}, T\right)$ from $\boldsymbol{\Phi}(T)$ via the matrix-vector product $\mathbf{\Psi}(T)=\mathbf{P} \Phi(T)$, as described in Sec. II, we set all components of $\boldsymbol{\Phi}(T)$ corresponding to atomic states below the DIT equal to zero. Therefore, the resulting $\boldsymbol{\Psi}(T)$ is a continuum wave function describing doubly ionized continua, as well as singly ionized continua with energies above the DIT. The radial integration in the domain $S_{3}$ is then used to approximately separate the doubly ionized continua from the remaining singly ionized continua.

Angular distributions for double ionization are obtained by omitting the integration over angles in Eq. (16). The remaining quantity, 


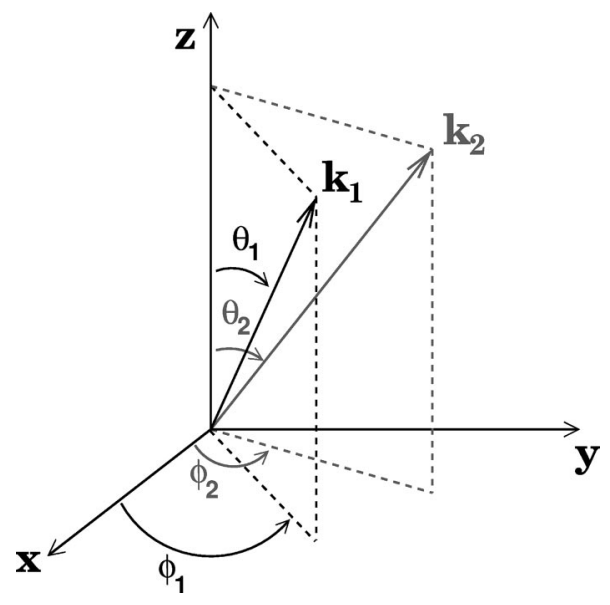

FIG. 10. Angular parametrization, in spherical coordinates, of the final-state ejection directions, $\mathbf{k}_{1}$ and $\mathbf{k}_{2}$, of the two continuum electrons. $\mathbf{k}_{1}$ is parametrized by the angles $\theta_{1}$ and $\phi_{1}$, and $\mathbf{k}_{2}$ by $\theta_{2}$ and $\phi_{2} . z$ denotes the laser polarization axis.

$$
\frac{d^{2} P}{d \Omega_{1} d \Omega_{2}}=\int_{r_{1}>r_{c}} d r_{1} \int_{r_{2}>r_{c}} d r_{2}\left|\Psi\left(\mathbf{r}_{1}, \mathbf{r}_{2}, T\right)\right|^{2},
$$

may be interpreted as a doubly differential double-ionization probability (DDDIP) for electron 1 to be ejected within the solid angle $d \Omega_{1}$ and electron 2 within $d \Omega_{2}$. The DDDIP is a function of the four spherical angles $\theta_{1}, \phi_{1}, \theta_{2}$, and $\phi_{2}$ (see Fig. 10), and thus is, in fact, fourfold differential in these angles. This parametrization in terms of spherical angles, as shown in Fig. 10, allows one to assess the role of the polarization axis $z$, since $\theta_{1}$ and $\theta_{2}$ represent the angles of the two electrons with respect to this axis. Once $\Psi\left(\mathbf{r}_{1}, \mathbf{r}_{2}, T\right)$ is obtained, the DDDIP can be evaluated for any combination of the four angles mentioned above, providing thereby complete information regarding the directions of ejection of the two electrons following double ionization. As the DDDIP is not differential in energy, it accounts for all possible energy transfers to the electrons from the laser pulse as well as for all possible energy-sharing distributions among the two electrons. Note that the above definition of the DDDIP - as well as its possible numerical evaluation-has been made possible due to the configuration interaction structure of the basis expansion (9), which allows for an implicit separation of radial and angular integrations in Eq. (16).

\section{B. DDDIP for the low-intensity (single-photon) case}

We first consider double ionization by a single photon, which is the dominant process at low laser intensity. Double ionization by a single photon has been widely studied, and the general features of the corresponding angular distributions are well established [53]. The purpose of this section is to show that our theory is able to reproduce most of these general features.

We consider a weak laser pulse of intensity $10^{9} \mathrm{~W} / \mathrm{cm}^{2}$ and frequency $\omega=0.235$ a.u. $(193.9 \mathrm{~nm}$ or $6.39 \mathrm{eV}$ photons), with 38 cycles within the FWHM (corresponding to about $24 \mathrm{fsec}$ ). The above frequency corresponds to about $0.4 \mathrm{eV}$ of excess energy above the DIT, and, because of the

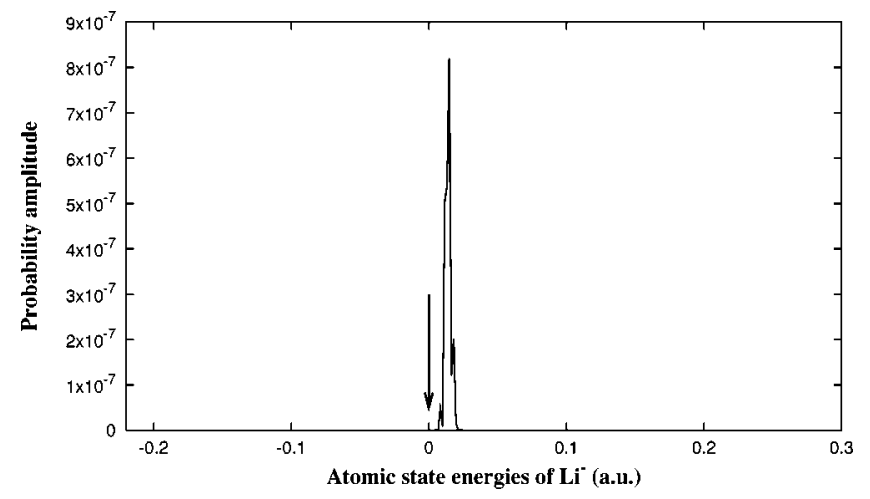

FIG. 11. Probability amplitude of the wave function at the end of the laser pulse with respect to the atomic energies of $\mathrm{Li}^{-}$(ground state excluded). The laser pulse has a peak intensity $I$ $=10^{9} \mathrm{~W} / \mathrm{cm}^{2}$, frequency $\omega=0.235$ a.u. $(193.9 \mathrm{~nm})$, and FWHM of $24 \mathrm{fsec}$. The arrow indicates the double-ionization threshold.

weak field intensity considered, single-photon absorption is the dominant process. Figure 11 gives a plot of the probability amplitude $\left|C_{n_{L}}\right|^{2}$ [see Eq. (13)] with respect to the atomic energy $E_{n}$ of the $n$th atomic basis state (ground state excluded), contained in the final wave function. The presence in Fig. 11 of a single peak right above the DIT (located at $E=0$ and indicated by the vertical arrow) is consistent with the absorption of a single photon. In addition, the population depleted from the ground state is almost identical to the population found around the single peak that appears in Fig. 11 above the DIT, thereby providing further evidence that single-photon absorption is the dominant process.

Figure 12 displays, in polar coordinates, the DDDIP for a coplanar emission of the two electrons. The polar plot in this figure represents the distribution of electron 2 , when electron 1 is ejected along the unit vector $\mathbf{k}_{1}$ at angles $\theta_{1}=0$ [Fig. 12(a)], $\theta_{1}=\pi / 4$ [Fig. 12(b)], $\theta_{1}=\pi / 2$ [Fig. 12(c)], and $\theta_{1}$ $=3 \pi / 4$ [Fig. 12(d)]. Figure 12 illustrates interesting features (a)

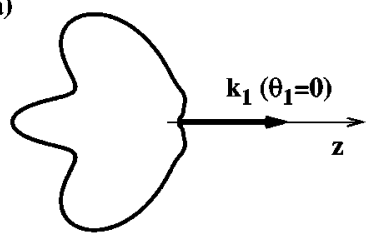

(c)

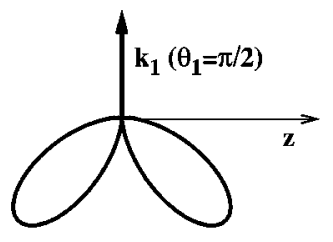

(b)

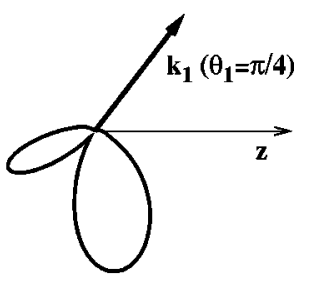

(d)

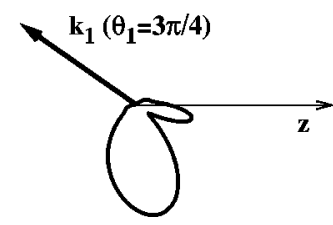

FIG. 12. DDDIP for single-photon double ionization (coplanar) of $\mathrm{Li}^{-}$by a weak, linearly polarized laser pulse of peak intensity $I=10^{9} \mathrm{~W} / \mathrm{cm}^{2}$, frequency $\omega=0.235$ a.u. $(193.9 \mathrm{~nm})$, and FWHM of about 24 fsec. $\mathbf{k}_{1}$ is a unit vector indicating the angle $\theta_{1}$ at which electron 1 is emitted with respect to the laser polarization axis $z$; the plot gives the distribution of the other electron. (a) $\theta_{1}=0$; (b) $\theta_{1}$ $=\pi / 4$; (c) $\theta_{1}=\pi / 2$; (d) $\theta_{1}=3 \pi / 4$. 
of the two ejected electrons: First, the probability for double ejection in opposite directions or at large relative angles is overwhelmingly larger than the probability for ejection in the same direction. Indeed, the two-electron ejection at zero relative angles is negligible; the two-electrons are predominantly ejected at large relative angles. Typically, if one electron (say electron 1) is ejected at a given angle in the direction $\mathbf{k}_{1}$, the distribution of electron 2 is significant only in the half plane opposite to the direction of $\mathbf{k}_{1}$. This feature stems from the Coulomb repulsion between the two electrons, which prevents them from being ejected at small relative angles. A second interesting feature shown in Fig. 12 is the fact that the polarization axis $z$ along which the linearly polarized field drives the two electrons does not play a major role in determining the characteristics of their angular distributions. Its direction influences primarily the relative magnitudes of the lobes giving the angular distribution of electron 2. These two features indicate that the low-intensity, single-photon double-ionization process is essentially dominated by electron-electron correlations. This fact has been established by TDCS calculations in the perturbative regime [14].

The lobe structures of the angular distributions warrant further comment. The fact that the angular distributions of electron 2 consist essentially of two lobes whose relative sizes depend on the angle between $\mathbf{k}_{1}$ and the $z$ axis is in agreement with perturbative calculations [54] and with a "Wannier" analysis [55]. An exception to this two-lobe structure is found in Fig. 12(a), which is an illustration of the fact that our angular distributions are not differential in energy, and thus account for configurations involving both equal and unequal sharing of the excess energy between the two electrons. Indeed, according to selection rules for the TDCS for the case of equal energy sharing [54], there should be nodes at the angles $\left(\theta_{1}, \theta_{2}\right)=(0, \pi)$ and $\left(\theta_{1}, \theta_{2}\right)=(\pi, 0)$, i.e., the lobe along the polarization axis in Fig. 12(a) should not exist. The appearance of this lobe in our results is due to contributions from configurations corresponding to an unequal energy sharing of the excess energy between the two electrons.

Finally, consider the node in Fig. 12(c) for $\theta_{2}= \pm \pi / 2$, which indicates that there is no ejection of both electrons in the direction perpendicular to the polarization axis. This is in agreement with a selection rule for the TDCS, which has been proved in Ref. [54] to be valid whatever the sharing of the excess energy between the two electrons. This selection rule is a consequence of the ${ }^{1} S^{e}$ and ${ }^{1} P^{o}$ symmetries of the initial and final states, respectively, as well as the dipole approximation and the linear polarization of the field.

We have thus shown that our results for the weak-field single-photon double ionization are consistent with perturbative cross-section calculations and selection rules. Differences from known perturbative results are understood as consequences of the finite pulse length. These comparisons therefore indicate the reliability of our technique for obtaining angular distributions for double ionization by ultrashort laser pulses.

\section{DDDIP for the high-intensity (multiphoton) case}

Having shown that our technique for obtaining angular distributions for double ionization yields results in agreement

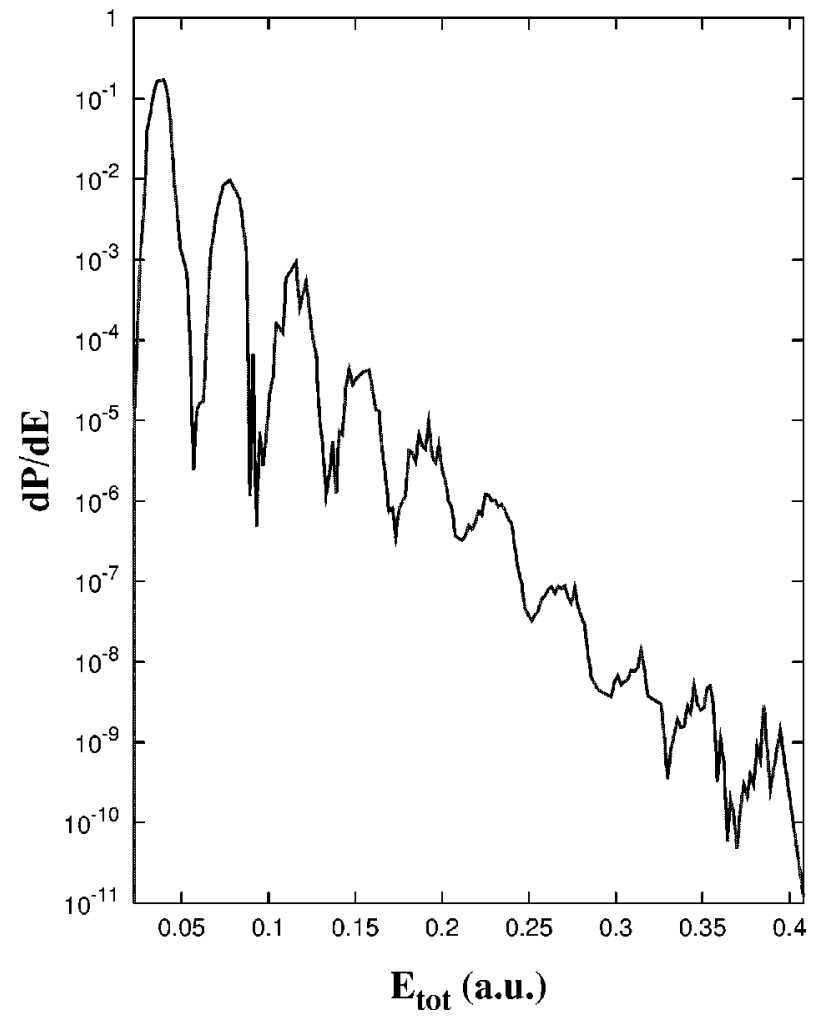

FIG. 13. ATD spectrum of $\mathrm{Li}^{-}$after interaction with an ultrashort, intense laser pulse of peak intensity $2 \times 10^{11} \mathrm{~W} / \mathrm{cm}^{2}$, frequency $\omega=0.038$ a.u. $(1199.0 \mathrm{~nm})$, and a FWHM of $12 \mathrm{fsec}$.

with perturbative calculations, we now apply it to a highintensity regime by considering an ultrashort laser pulse having the following characteristics: Intensity $I=2$ $\times 10^{11} \mathrm{~W} / \mathrm{cm}^{2}$, frequency $\omega=0.038$ a.u. $(1199.0 \mathrm{~nm})$, pulse duration consisting of three laser periods within FWHM (12 fsec.). This photon frequency is above the single-ionization threshold of $\mathrm{Li}^{-}$, and about six photons are necessary to reach the DIT. (Our choice of this frequency is dictated by the limitations of our computer resources: For example, a much lower frequency would require a larger number of photons to doubly ionize $\mathrm{Li}^{-}$, and the corresponding simulation would require more angular momenta in the basis, which also means longer computation times and larger computer memory requirements.) Figure 13 displays the distribution of the total energy of the two electrons at the end of the laser pulse. As expected, this ATD spectrum shows a series of peaks, separated by the photon energy, which extends from the energy region just above the single-ionization threshold to energies above the double-ionization threshold.

Although we are able to evaluate the DDDIP for any combination of the four spherical angles mentioned above, we need to fix two of these angles in order to present 3D plots of our results for the DDDIP as a function of the other two angles. Therefore, in presenting results below, we make two choices of azimuthal angles $\phi_{1}$ and $\phi_{2}:\left(\phi_{1}=\phi_{2}=0\right)$ and $\left(\phi_{1}=0, \phi_{2}=\pi\right)$, both of which correspond to the case of coplanar emission of the two electrons. The laser polarization axis divides the emission plane in question into two half planes. In the case $\left(\phi_{1}=\phi_{2}=0\right)$, a plot of the DDDIP with 

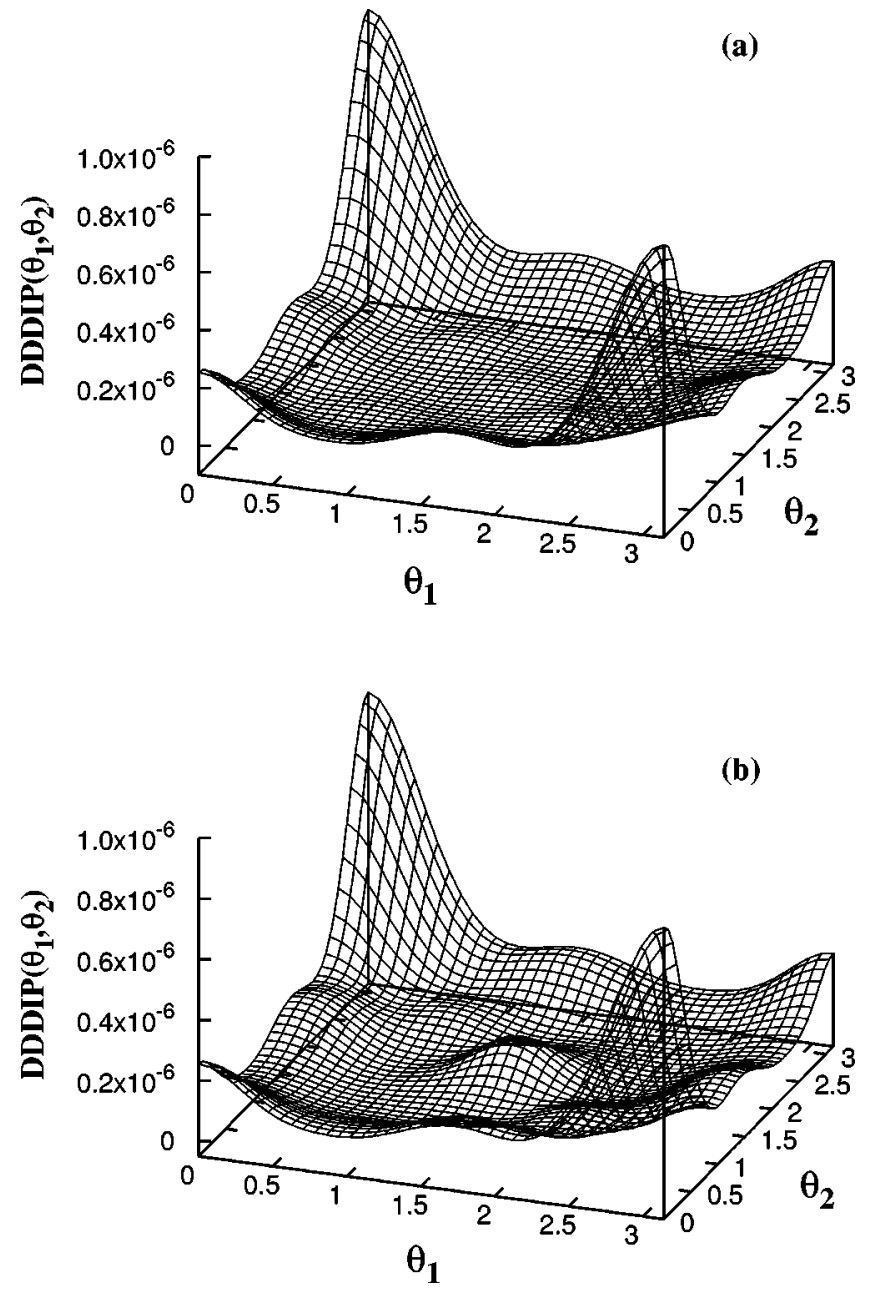

FIG. 14. Angular distributions for multiphoton double ionization of $\mathrm{Li}^{-}$by an ultrashort, intense laser pulse of peak intensity $2 \times 10^{11} \mathrm{~W} / \mathrm{cm}^{2}, \omega=0.038$ a.u. $(1199.0 \mathrm{~nm})$, and FWHM of 12 fsec. The doubly differential double-ionization probability (DDDIP) is plotted as a function of the angular positions of the ejected electrons (cf. Fig. 10) in two coplanar cases: (a) for $\left(\phi_{1}=0, \phi_{2}=0\right)$; (b) for $\left(\phi_{1}=0, \phi_{2}=\pi\right)$.

respect to the polar angles $\theta_{1} \quad\left(0 \leqslant \theta_{1} \leqslant \pi\right)$ and $\theta_{2} \quad\left(0 \leqslant \theta_{2}\right.$ $\leqslant \pi$ ) corresponds to the double ejection of the two electrons in the same half plane. The case $\left(\phi_{1}=0, \phi_{2}=\pi\right)$ corresponds to the case of double ejection of the two electrons in opposite half planes (i.e., electron 1 ejected in one half plane and electron 2 in the other half plane).

Three-dimensional plots of the DDDIP with respect to the polar angles $\left(\theta_{1}, \theta_{2}\right)$ are displayed in Fig. 14(a) for azimuthal angles $\left(\phi_{1}=0, \phi_{2}=0\right)$, and in Fig. 14(b) for azimuthal angles $\left(\phi_{1}=0, \phi_{2}=\pi\right)$. Figure 14 shows novel features not observed in the previously considered singlephoton case: The two electrons may now be ejected in all directions, indicating an apparent breakdown of the weakfield selection rules. In particular, we find that not only may both electrons be ejected with zero relative angles, but also both electrons may be ejected in the direction perpendicular to the polarization axis. In addition, the polarization axis plays a significant role, as electrons are now predominantly ejected along this axis. Indeed, the DDDIP in Fig. 14 shows (a)

(c)

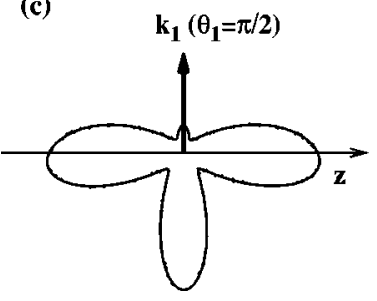

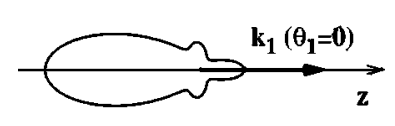

(b)

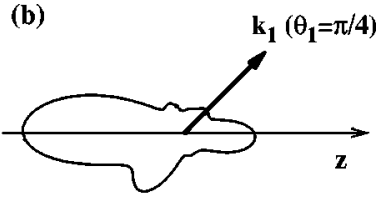

(d)

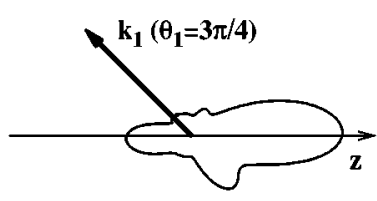

FIG. 15. Plots in polar coordinates of the angular distributions for multiphoton double ionization of $\mathrm{Li}^{-}$by an ultrashort, intense laser pulse with the same parameters as in Fig. 14. The polar plot represents the distribution of electron 2, when electron 1 is ejected along the unit vector $\mathbf{k}_{1}$ at various angles $\theta_{1}$ relative to the polarization axis $z$ : (a) $\theta_{1}=0$; (b) $\theta_{1}=\pi / 4$; (c) $\theta_{1}=\pi / 2$; (d) $\theta_{1}=3 \pi / 4$;

four prominent peaks corresponding to two-electron ejection in the four possible configurations along the $z$ axis: (i) both along positive $z$ (i.e., peaks located in the vicinity of $\theta_{1}$ $=\theta_{2}=0$ in Fig. 14); (ii) both along negative $z$ (i.e., peaks located in the vicinity of $\theta_{1}=\theta_{2}=\pi$ in Fig. 14); (iii) electron 1 along positive $z$ and electron 2 along negative $z$ (i.e., peaks located in the vicinity of $\theta_{1}=0, \theta_{2}=\pi$ in Fig. 14); (iv) electron 2 along positive $z$ and electron 1 along negative $z$ (i.e., peaks located in the vicinity of $\theta_{1}=\pi, \theta_{2}=0$ in Fig. 14). The configurations (iii) and (iv) are identical in magnitude, as they are symmetric under exchange of the two electrons. Therefore, for an intense laser, the polarization axis along which the field drives electrons becomes highly relevant. Also, because of the intensity of the field, it is now possible for two electrons to be ejected at zero relative angle along the polarization axis. However, the DDDIP for doubleelectron ejection in opposite directions along the $z$ axis is larger in magnitude than that corresponding to ejection in the same direction (zero relative angle) along this axis-an indication that electron-electron correlations still influence the double-ejection process. These features are better illustrated in Fig. 15, which is to be contrasted to Fig. 12 for the weakfield, single-photon case. It shows plots in polar coordinates of the DDDIP for electron 2, when electron 1 is ejected along the unit vector $\mathbf{k}_{1}$. Figure 15(a) shows that when electron 1 is ejected along the $z$ axis $\left(\theta_{1}=0\right)$, electron 2 is ejected predominantly in the opposite direction, but there is also a smaller but significant probability that electron 2 is ejected in the same direction as electron 1. In general and in stark contrast to the weak-field case (cf. Fig. 12), one sees in Fig. 15 that no matter which direction $\mathbf{k}_{1}$ one selects for ejection of electron 1 , there is always a large probability for ejection of electron 2 along the laser polarization axis, in both positive and negative directions. This is a clear illustration of the importance of the laser field and its polarization axis for high intensities. However, the fact that the relative magnitudes of the DDDIP for ejection of electron 2 along the positive and 
the negative directions of the polarization axis depends on the direction of ejection of electron 1 is an indication that the influence of correlations (Coulomb repulsion) on the doubleionization process is also significant. Indeed, the plots in Fig. 15 show that the DDDIP for electron 2 is always larger on the side of the $x-y$ plane (i.e., the plane perpendicular to the $z$ axis at the origin) where electron 1 does not appear.

There is an additional interesting feature in Figs. 14 and 15(c) that we have not yet discussed. This feature appears in Fig. 14(b) as a local maximum at the angles $\theta_{1}=\theta_{2}=\pi / 2$, which correspond to double ejection of both electrons in opposite directions perpendicularly to the polarization axis. A similar maximum is also present, but barely visible, in Fig. 14(a) at the same angles; it corresponds to double ejection of the two electrons in the same direction (with zero relative angle) perpendicularly to the polarization axis. Figure 15(c) gives a better illustration of both cases. It follows that in the high-intensity (multiphoton) regime, the two electrons may be ejected in directions perpendicular to the polarization axis, contrary to the low-intensity (single photon) regime, where this is impossible. As shown in Fig. 15(c) this double ejection perpendicular to the $z$ axis is more intense in the configuration where the two electrons are ejected in opposite directions than for the configuration where they are ejected in the same direction. This is another signature of correlation effects.

In order to further investigate double ejection perpendicular to the $z$ axis, we have performed an angular-momentumbased analysis of the DDDIP. Specifically, starting from the final wave function $\Psi(T)$ (from which the remaining population of the ground state as well as the populations of the doubly excited states and the singly excited states below the double-ionization threshold have been removed, as discussed in Sec. V A), we have considered the following two cases.

(i) We have evaluated the DDDIP by using the portion of $\Psi(T)$ from which all even total angular-momentum components $[L=0,2,4,6,8$; cf. Eq. (9) $]$ have been excluded (i.e., set equal to zero), so that the resulting $\Psi(T)$ [denoted hereafter $\left.\Psi^{o}(T)\right]$ only contains odd $L$ components $(L=1,3,5,7)$ and therefore (for the case of linearly polarized light) has an odd parity, $(-1)^{L}$. The corresponding DDDIP for electron 2 for the case in which electron 1 is ejected at the angle $\theta_{1}=\pi / 2$ is plotted in the top panel of Fig. 16 in both Cartesian and polar coordinates. These results show a node at $\theta_{2}= \pm \pi / 2$, which indicates that there is no ejection of both electrons perpendicular to the polarization axis.

(ii) In the second case considered, we have evaluated the DDDIP with $\Psi(T)$ from which all odd $L$ components [cf. Eq. (9)] have been excluded (i.e., set equal to zero), so that the resulting $\Psi(T)$ [denoted hereafter $\Psi^{e}(T)$ ] contains only even $L$ components, and thus (again, for the case of linearly polarized light) has an even parity. The corresponding DDDIP for electron 2 when electron 1 is being ejected at the angle $\theta_{1}=\pi / 2$ are plotted in the bottom panel of Fig. 16 in both Cartesian and polar coordinates. These results show that there are maxima at $\theta_{2}= \pm \pi / 2$, indicating that both electrons are ejected perpendicularly to the laser polarization axis.
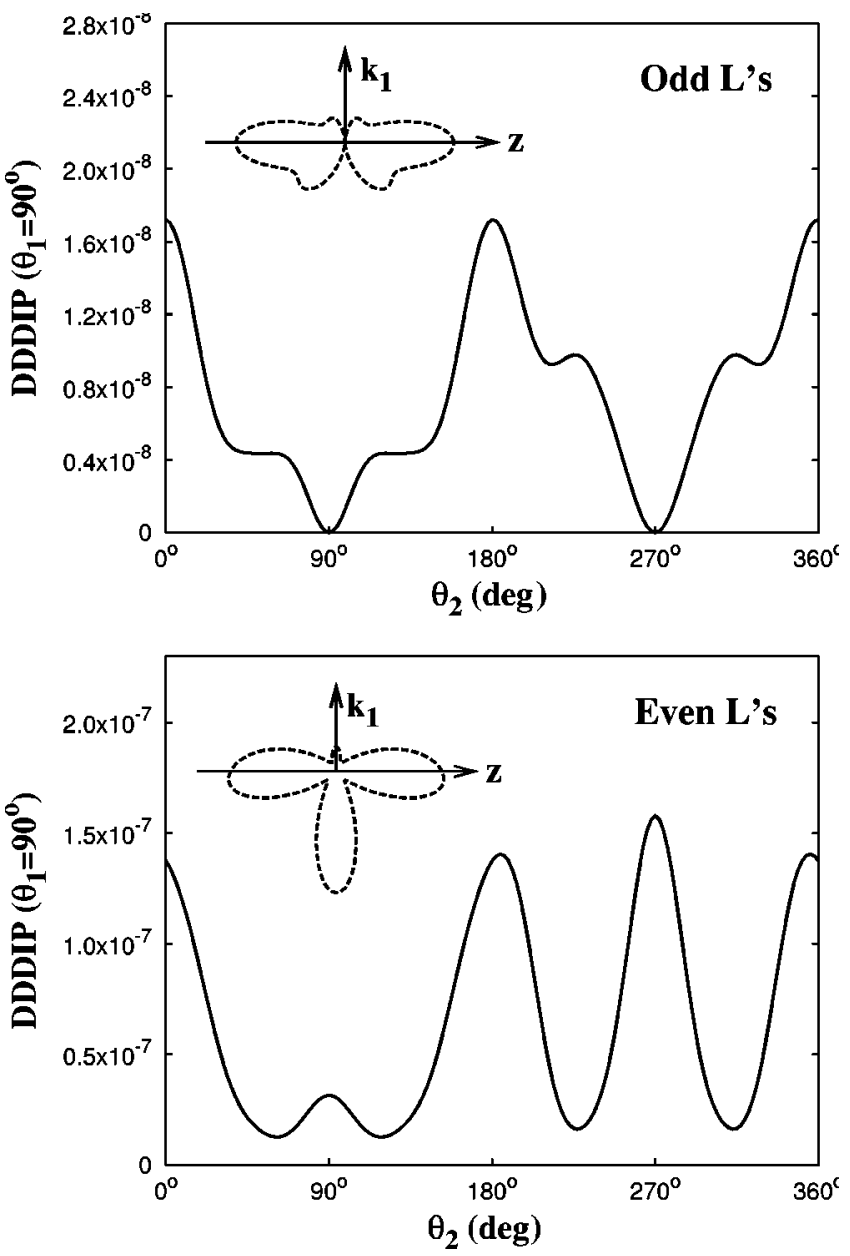

FIG. 16. Odd and even parity contributions to the DDDIP for electron 2 when electron 1 is ejected perpendicularly to the laser polarization axis (i.e., $\theta_{1}=\pi / 2$ ). Angular distributions are obtained by considering only odd (top figure) or only even (bottom figure) angular momentum $L$ contributions to the final wave function. The inset in each figure is the corresponding plot in polar coordinates. The laser pulse has the same parameters as in Fig. 14.

As $\Psi(T)=\Psi^{o}(T)+\Psi^{e}(T)$, it follows that coplanar double ejection of both electrons in directions perpendicular to the laser polarization axis arise only from even $L$ components of the wave function. This fact is further supported by Figs. 17, 18, and 19 that show the DDDIP obtained for selected single- $L$ components of $\Psi(T)$. Here again, one sees that when only odd $L$ components of the wave function are considered, the resulting DDDIPs show no double ejection perpendicularly to the polarization axis, whereas when only even $L$ 's are considered, the DDDIPs show a local maximum for this double-ejection configuration. In the dipole approximation, an odd- $L$ channel is only populated by the absorption of an odd number of photons, whereas an even- $L$ channel is only populated following absorption of an even number of photons (starting from the ground state with $L$ $=0$ ). Therefore, we may conclude that ejection of both electrons perpendicularly to the laser polarization axis is only due to an absorption of an even number of photons, and that for double ionization following absorption of an odd number of photons, the two electrons cannot both be ejected in di- 

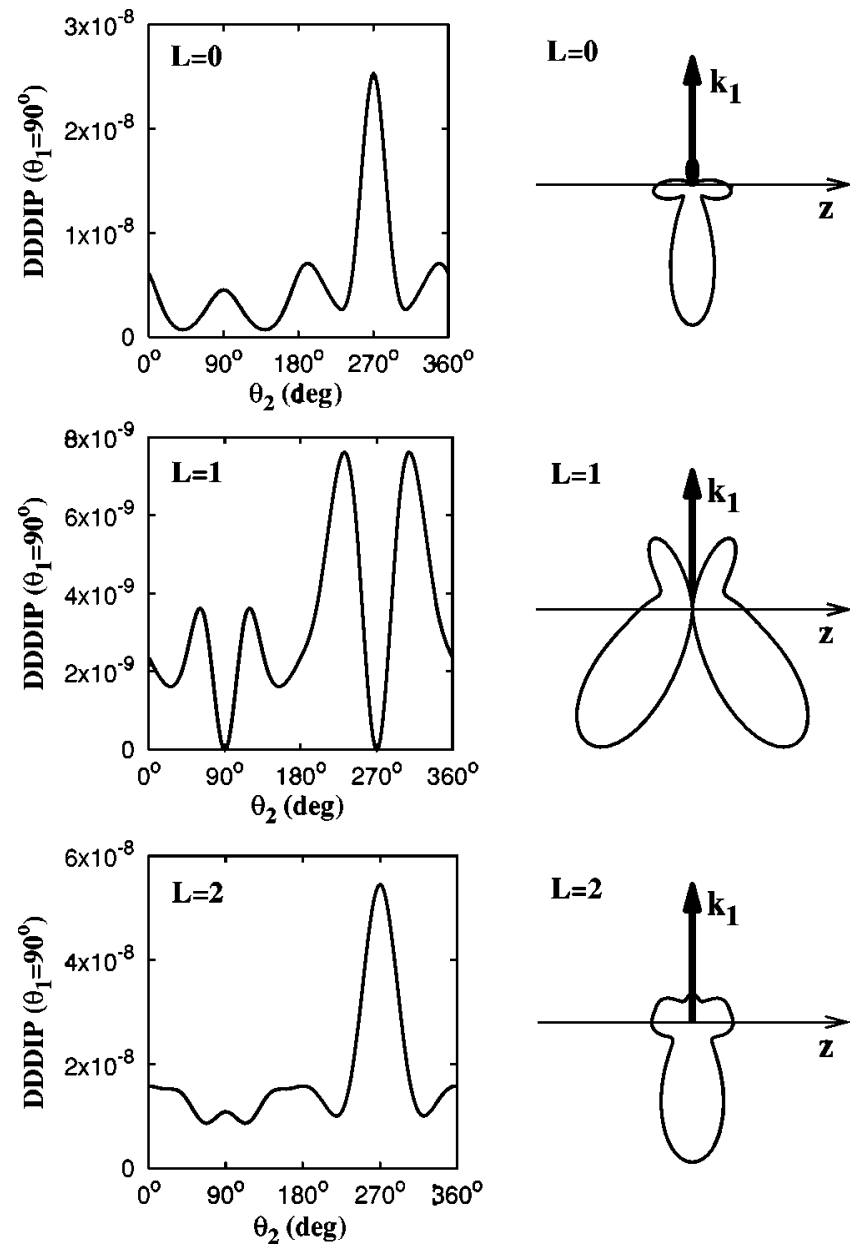

FIG. 17. DDDIP for electron 2, for the case in which electron 1 is ejected in the direction perpendicular to the laser polarization axis (i.e., $\theta_{1}=90^{\circ}$ ). For each row of plots, the DDDIP is obtained by using a final wave function where only components corresponding to a specific single total angular momentum $L$ are kept: $L=0$ (top row), $L=1$ (middle row), $L=2$ (bottom row). For each row, the left and right plots are respectively in Cartesian and polar coordinates. Double ejection of both electrons perpendicularly to the laser polarization axis occurs only for even values of $L$.

rections perpendicular to the polarization axis. In fact, the case of single-photon double ionization discussed in Sec. $\mathrm{V} \mathrm{B}$ is an illustration of this rule. The present results extend to the case of absorption of an arbitrary odd number of photons the selection rule (derived in Ref. [54] for single-photon double ionization) that excludes double ejection of both electrons perpendicularly to the laser polarization axis. This shows that exchange, parity, and angular momentum symmetry considerations affect the angular distributions of multiphoton double ionization.

Note that the DDDIP does not consist only of individual contributions from the odd and even components of the wave function. In fact there are, of course, contributions also due to interferences between the odd and even components [as can be seen by substituting the square norm of $\Psi(T)$ $=\Psi^{o}(T)+\Psi^{e}(T)$ into Eq. (17)]. Therefore, the net maximum in the DDDIP in the direction perpendicular to the polarization axis is only obtained when these three contribu-
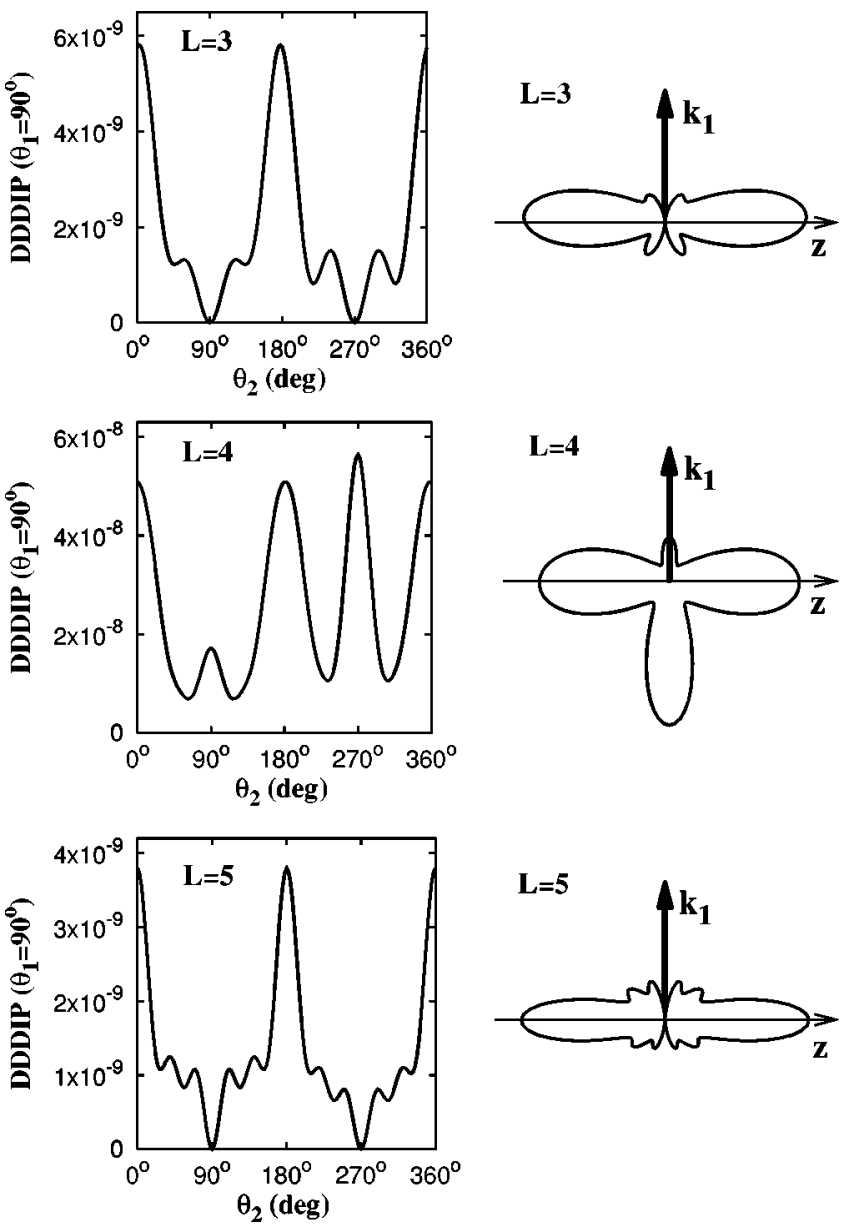

FIG. 18. Same as Fig 17 for angular momenta $L=3$ (top row), $L=4$ (middle row), and $L=5$ (bottom row). Double ejection of both electrons perpendicularly to the laser polarization axis occurs only for even values of $L$.

tions interfere constructively so as to generate such a maximum.

\section{SUMMARY AND CONCLUSIONS}

In conclusion, we have developed a configuration interaction approach for a direct numerical solution of the TDSE for multielectron systems interacting with an ultrashort, intense, and linearly polarized laser field, in the two-active-electron (TAE) approximation. The method is nonperturbative, three dimensional, and accounts for electron-electron and electroncore interactions, as well as for the polarization of the core by the active electrons. This approach is applicable to atomic systems consisting of a core (comprising the nucleus and inner shell electrons) and two-active electrons. By adjusting the semiempirical parameters in the core potential, this approach is applicable to any atomic systems having two valence electrons outside closed shells.

A technique for obtaining angular distributions for double ionization by an ultrashort laser pulse has also been discussed. This technique allows one to obtain detailed information about the directions of ejection of the two electrons for both weak and intense fields, while excluding spurious contributions that may arise from the population left at the end of time propagation (i.e., after the passage of the laser 

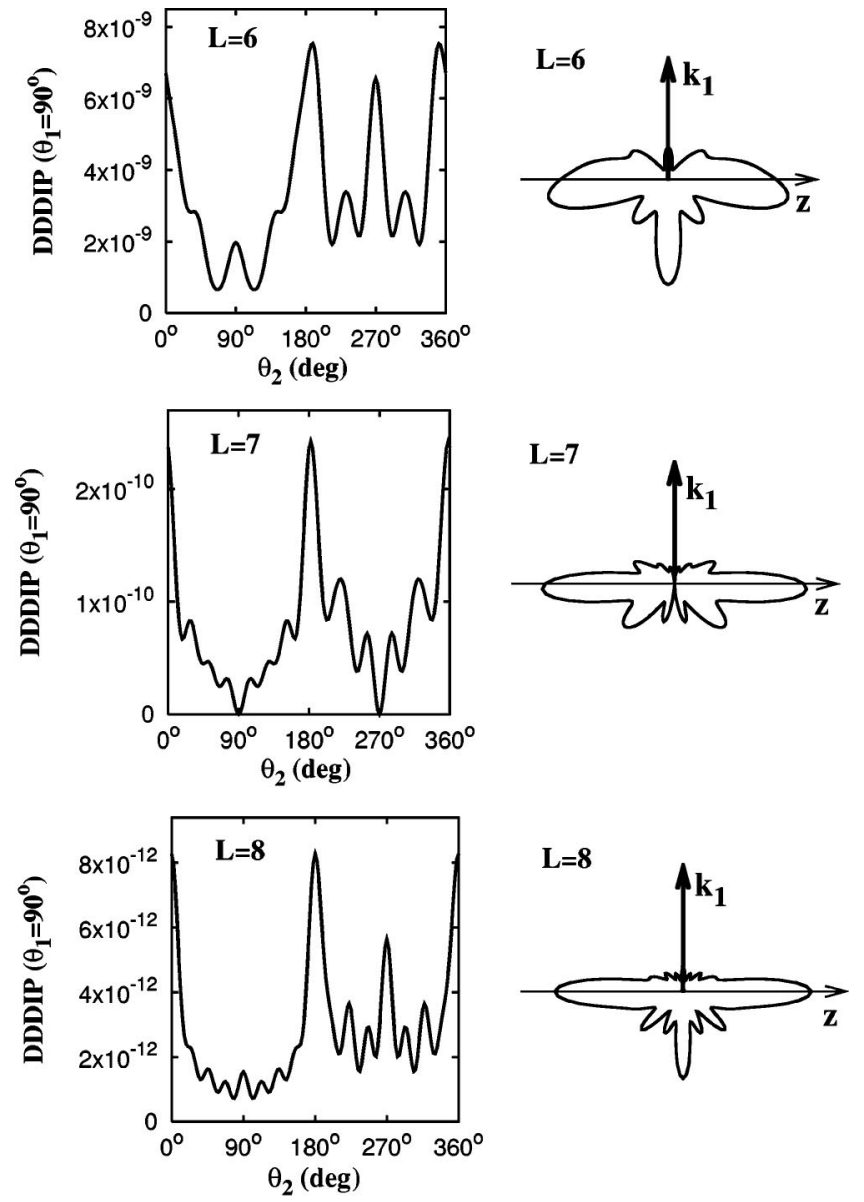

FIG. 19. Same as Fig 17 for angular momenta $L=6$ (top row), $L=7$ (middle row), and $L=8$ (bottom row). Double ejection of both electrons perpendicularly to the laser polarization axis occurs only for even values of $L$.

pulse) in atomic states below the double-ionization threshold.

We have used the above numerical approach to study the photodetachment of $\mathrm{Li}^{-}$by ultrashort laser pulses. For weak field intensities, our results for the detachment yield agree with known features of lowest-order-perturbation theory (LOPT). Indeed, plots on a log-log scale of the detachment yield with respect to the laser peak intensity show linear behaviors at low intensity, with slopes that agree with expectations from LOPT. For the laser frequency $\omega=0.024$ a.u., which is just above the detachment threshold, we find that the intensity-dependent detachment yield shows an inflection that is consistent with the closure of the one-photon detachment channel (due to ac Stark and ponderomotive shifts) and with the increasing importance of the two-photon channel. An analysis of the photoelectron energy distribution confirms the interpretation of this inflection as due to the closure of the one-photon channel and the rise of the two-photon chan- nel. In addition, and despite the presence of a pulse in our calculations, a comparison of our results with $\mathcal{R}$-matrix Floquet detachment rates integrated over the pulse envelope employed in this work shows a satisfactory agreement. We have also displayed the dynamics of the photodetachment process via the time evolution of the ground state and of the Coulomb repulsion between the two electrons, as well as the angle-integrated radial probability distributions of the finalstate wave functions. The time evolution of the ground state indicates that the nonperturbative behavior of $\mathrm{Li}^{-}$sets in at relatively low intensities (of the order of $10^{10} \mathrm{~W} / \mathrm{cm}^{2}$ ).

Finally, we have obtained angular distributions for double ionization of $\mathrm{Li}^{-}$by ultrashort laser pulses, for both the weak-intensity (single photon) and the high-intensity (multiphoton) cases. For the first case, we have reproduced essential features (such as the eminent importance of electronelectron correlations, selection rules, etc.) already established for single-photon double ionization. For the second case, we find that the two electrons are predominantly ejected along the laser polarization axis in the same direction (zero relative angle) and in opposite directions ( $180^{\circ}$ relative angle). In addition, for the multiphoton case (in contrast to the singlephoton case), electrons may be ejected perpendicularly to the laser polarization axis, both in the same direction (at zero relative angle) and in opposite directions $\left(180^{\circ}\right.$ relative angle). We have shown that an ejection of both electrons perpendicularly to the laser polarization axis arises from the even angular-momentum components of the wave function, as odd angular momenta do not contribute to double ionization for this configuration. In other words, in the dipole approximation and within the framework of $L-S$ coupling, ejection of both electrons perpendicularly to the polarization axis (starting from an initial ${ }^{1} S^{e}$ state and for the case of linearly polarized light) only occurs following absorption of an even number of photons. Thus this study of angular distributions shows evidence of the continuing influence of electronelectron correlations and of wave-function symmetry properties on the directions of ejection of the two electrons following multiphoton double ionization by an ultrashort, intense laser pulse.

Note added in proof. We have recently become aware of LOPT calculations for two-photon double ionization of $\mathrm{He}$ for $\omega \approx 45 \mathrm{eV}$ by Makris, Nikolopoulos, and Lambropoulos [56]. Angular distributions for the case in which one electron is ejected along the laser polarization axis are analyzed. This work also shows that electrons can be emitted with zero relative angle along the polarization axis.

\section{ACKNOWLEDGMENTS}

We thank Chien-Nan Liu for fruitful discussions and D. H. Glass for providing us with his $\mathcal{R}$-matrix results. This work was supported by the U.S. Department of Energy, Office of Basic Energy Sciences, Division of Chemical Sciences, under Grant No. DE-FG03-96ER14646. 
[1] C.J. Joachain, M. Dörr, and N. Kylstra, Adv. At., Mol., Opt. Phys. 42, 225 (2000).

[2] P. Lambropoulos, P. Maragakis, and J. Zhang, Phys. Rep. 305, 203 (1998).

[3] J. Parker, K.T. Taylor, C.W. Clark, and S. Blodget-Ford, J. Phys. B 29, L33 (1996).

[4] A. Scrinzi and B. Piraux, Phys. Rev. A 58, 1310 (1998); 56, R13 (1997).

[5] M.S. Pindzola and F. Robicheaux, Phys. Rev. A 57, 318 (1998).

[6] L.A.A. Nikolopoulos and P. Lambropoulos, Phys. Rev. Lett. 82, 3771 (1999).

[7] G. Lagmago Kamta, Th. Grosges, B. Piraux, R. Hasbani, E. Cormier, and H. Bachau, J. Phys. B 34, 857 (2001).

[8] R. Hasbani, E. Cormier, and H. Bachau, J. Phys. B 33, 2101 (2000).

[9] K.C. Kulander, K.J. Schafer, and J.L. Krause, Adv. At., Mol., Opt. Phys. 1, 247 (1992).

[10] M.J. Nandor, M.A. Walker, L.D. Van Woerkom, and H.G. Muller, Phys. Rev. A 60, R1771 (1999).

[11] D.N. Fittinghoff, P.R. Bolton, B. Chang, and K.C. Kulander, Phys. Rev. Lett. 69, 2642 (1992).

[12] B. Walker, E. Mevel, B. Yang, P. Breger, J.P. Chambaret, A. Antonetti, L.F. DiMauro, and P. Agostini, Phys. Rev. A 48, R894 (1993).

[13] Th. Weber et al., Phys. Rev. Lett. 84, 443 (2000); R. Moshammer et al., ibid. 84, 447 (2000).

[14] J.S. Briggs and V. Schmidt, J. Phys. B 33, R1 (2000); G.C. King and L. Avaldi, ibid. 33, R215 (2000).

[15] L. Malegat, P. Selles, and A. Kazansky, Phys. Rev. A 60, 3667 (1999); A.S. Kheifets and I. Bray, J. Phys. B 31, L447 (1998); M. Pont and R. Shakeshaft, Phys. Rev. A 51, R2676 (1995).

[16] B. Witzel, N.A. Papadogiannis, and D. Charalambidis, Phys. Rev. Lett. 85, 2268 (2000).

[17] J.S. Parker, L.R. Moore, D. Dundas, and K.T. Taylor, in Multiphoton Processes, edited by Louis F. DiMauro, Richard R. Freeman, and Kenneth Kulander, AIP Conf. Proc. No. 525 (AIP, Melville, NY, 2000), p. 273.

[18] G. Lagmago Kamta and A.F. Starace, Phys. Rev. Lett. 86, 5687 (2001)

[19] G. Lagmago Kamta and A.F. Starace, in Super-Intense LaserAtom Physics, Vol. 12 of NATO Science Series II, edited by B. Piraux and K. Rza̧żewski (Kluwer, Dordrecht, The Netherlands, 2001), p. 143.

[20] Y.K. Bae and J.R. Peterson, Phys. Rev. A 32, 1917 (1985).

[21] J. Dellwo, Y. Liu, C.Y. Tang, D.J. Pegg, and G.D. Alton, Phys. Rev. A 46, 3924 (1992).

[22] U. Berzinsh, G. Haeffler, D. Hanstorp, A. Klinkmüller, E. Lindroth, U. Ljungblad, and D.J. Pegg, Phys. Rev. Lett. 74, 4795 (1995).

[23] U. Ljungblad, D. Hanstorp, U. Berzinsh, and D.J. Pegg, Phys. Rev. Lett. 77, 3751 (1996).

[24] G. Haeffler, I.Yu. Kiyan, D. Hanstorp, and D.J. Pegg, Phys. Rev. A 57, 2216 (1998).

[25] D.L. Moores and D.W. Norcross, Phys. Rev. A 10, 1646 (1974).

[26] R. Moccia and P. Spizzo, J. Phys. B 23, 3557 (1990).
[27] C.A. Ramsbottom, K.L. Bell, and K.A. Berrington, J. Phys. B 27, 2905 (1994).

[28] C. Pan, A.F. Starace, and C.H. Greene, Phys. Rev. A 53, 840 (1996).

[29] C.N. Liu and A. F. Starace, Phys. Rev. A 58, 4997 (1998).

[30] E. Lindroth, Phys. Rev. A 52, 2737 (1995).

[31] Th. Mercouris, Y. Komninos, S. Dionissopoulou, and C.A. Nicolaides, Phys. Rev. A 50, 4109 (1994).

[32] D.H. Glass, P.G. Burke, C.J. Noble, and G.B. Wöste, J. Phys. B 31, L667 (1998).

[33] D.H. Glass (private communication).

[34] F. Robicheaux and C.H. Greene, Phys. Rev. A 46, 3821 (1992).

[35] W.R. Johnson, D. Kolb, and K.N. Huang, At. Data Nucl. Data Tables 28, 333 (1983).

[36] C.E. Moore, Atomic Energy Levels, Natl. Bur. Stand. (U.S.) Circ. No. NSRDS-NBS35 (U.S. GPO, Washington, DC, 1971).

[37] D.A. Varshalovich, A.N. Moskalev, and V.K. Khersonskii, Quantum Theory of Angular Momentum (World Scientific, Singapore, 1988).

[38] See, e.g., L. Lapidus and J.H. Seinfeld, Numerical Solution of Ordinary Differential Equations (Academic Press, New York, 1971).

[39] See, e.g., I.A. Dodes, Numerical Analysis for Computer Science (North-Holland, New York, 1978).

[40] One may think that we assume that electrons are distinguishable, which is not true. In fact, applying the symmetrization "restores" their indistinguishability.

[41] G. Lagmago Kamta, B. Piraux, and A. Scrinzi, Phys. Rev. A 63, 040502(R) (2001).

[42] B. Piraux and G. Lagmago Kamta, in Super-Intense LaserAtom Physics (Ref. [19]), p. 127.

[43] E. Hairer, S.P. Nørsett, and G. Wanner, Solving Ordinary Differential Equations I: Nonstiff Problems (Springer-Verlag, Berlin, 1987).

[44] K.T. Chung and P. Fullbright, Phys. Scr. 45, 445 (1992).

[45] W.E. Lamb, Jr., R.R. Schlicher, and M.O. Scully, Phys. Rev. A 36, 2763 (1987).

[46] P. Lambropoulos, M.A. Kornberg, L.A.A. Nikolopoulos, and A. Saenz, in Multiphoton Processes (Ref. [17]), p. 231.

[47] R.R. Freeman, P.H. Bucksbaum, H. Milchberg, S. Darack, D. Schumacher, and M.E. Geusic, Phys. Rev. Lett. 59, 1092 (1987).

[48] H.G. Muller et al., Phys. Rev. Lett. 60, 565 (1988).

[49] J.H. Eberly and J. Javanainen, Phys. Rev. Lett. 60, 1346 (1988).

[50] L.A. Collins and A.L. Merts, Phys. Rev. A 37, 2415 (1988).

[51] J.N. Bardsley, A. Szöke, and M.J. Comella, J. Phys. B 21, 3899 (1988).

[52] D. Dundas, K.T. Taylor, J.S. Parker, and E.S. Smyth, J. Phys. B 32, L231 (1999); D.G. Lappas, P.P. Corso, and P.L. Knight, in Multiphoton Processes (Ref. [17]), p. 245; H.G. Muller, ibid., p. 257; R. Panfili, C. Szymanowski, W.C. Liu, and J.H. Eberly, ibid., p. 265.

[53] J.S. Briggs and V. Schmidt, J. Phys. B 33, R1 (2000); G.C. King and L. Avaldi, ibid. 33, R215 (2000).

[54] F. Maulbetsch and J.S. Briggs, J. Phys. B 26, 1679 (1993).

[55] A. Huetz, P. Selles, D. Waymel, and J. Mazeau, J. Phys. B 24, 1917 (1991).

[56] M.G. Makris, L.A.A. Nikolopoulos, and P. Lambropoulos, Europhys. Lett. 54, 722 (2001). 